\title{
QUASIANALYTIC CARLEMAN CLASSES ON BOUNDED DOMAINS
}

\author{
K. V. TRUNOV AND R. S. YULMUKHAMETOV
}

\begin{abstract}
Several criteria for the quasianaliticity of Carleman classes at a boundary point of a Jordan domain with rectifiable boundary are found.
\end{abstract}

\section{INTRODUCTION}

We start with recalling the necessary definitions from the paper 1 .

Let $E$ be a perfect compact set in the plane $\mathbb{C}$. A complex-valued function $f$ is said to be infinitely differentiable on $E$ if there exist functions $f_{0}, f_{1}, \ldots$ continuous on $E$ with $f_{0}(z) \equiv f(z), z \in E$, and such that, for any $n=0,1,2, \ldots, k=0,1, \ldots, n$, the functions

$$
R_{n, k}(\zeta, z):=f_{k}(\zeta)-\sum_{p=0}^{n-k} f_{k+p}(z) \frac{(\zeta-z)^{p}}{p !}
$$

satisfy the estimate

$$
\left|R_{n, k}(\zeta, z)\right|=o\left(|\zeta-z|^{n-k}\right)
$$

uniformly in $\zeta, z \in E$. Note that for any infinitely differentiable function $f$ the functions $f_{k}$ are determined uniquely by $f$ via the recurrence relations

$$
f_{0}(z)=f(z), \quad f_{k+1}(z)=\lim _{\zeta \longrightarrow z} \frac{f_{k}(\zeta)-f(z)}{\zeta-z}, \quad k=0,1, \ldots
$$

In particular, the function $f$ turns out to be holomorphic at the interior points of $E$ and, moreover, $f_{k}(z)=f^{(k)}(z)$, and the derivatives of $f$ extend continuously up to the boundary of the set $E$. Having this in mind, in what follows we write $f^{(k)}$ in place of $f_{k}$ for the functions infinitely differentiable on $E$.

For an increasing sequence of positive numbers $\mathcal{M}=\left(M_{n}\right)_{n=0}^{\infty}$ and for a positive integer $q$, we denote by $A_{q}(E, \mathcal{M})$ the class of functions $f$ infinitely differentiable on $E$ and satisfying the condition

$$
\left|R_{n, k}(\zeta, z)\right| \leq C_{f} q^{n+1} M_{n+1} \frac{|\zeta-z|^{n-k+1}}{(n-k+1) !}, \quad \zeta, z \in E,
$$

where the constant $C_{f}$ depends neither on $n, k$ nor on $\zeta, z \in E$. The Carleman class $A(E, \mathcal{M})$ is defined as the union of all classes $A_{q}(E, \mathcal{M}), q \in \mathbb{N}$.

If $E$ is a closed interval $I$ of the real axis, then, by the Taylor formula, the Carleman classes can be described in a classical way as the classes of those infinitely differentiable functions $f$ on the corresponding open interval $I^{0}$ for which there exist $q_{f} \in \mathbb{N}$ and $C_{f}>0$ such that

$$
\left|f^{(k)}(x)\right| \leq C_{f} q_{f}^{k} M_{k}, \quad k=0,1, \ldots, x \in I^{0} .
$$

2000 Mathematics Subject Classification. Primary 30D60.

Key words and phrases. Carleman classes, quasianaliticity.

Supported by RFBR (grant no. 06-01-00516-a). 
In the present paper the set $E$ will be the closure of a simply connected bounded domain $D$ in $\mathbb{C}$ with rectifiable Jordan boundary. In this case, the functions of class $A(\bar{D}, \mathcal{M})$ are holomorphic in $D$ and admit continuous extensions to the boundary of $D$ together with their derivatives. In what follows, we denote by $f^{(k)}(z)$ the $k$ th order derivative of $f$ extended continuously to the boundary of $D$. Thus, the class $A(\bar{D}, \mathcal{M})$ consists of functions $f$ holomorphic in $D$ and satisfying the condition

$$
\sup _{n \geq 0, k \leq n} \sup _{z, \zeta \in D} \frac{(n-k+1) !}{q^{n+1} M_{n+1}|\zeta-z|^{n-k+1}}\left|f^{(k)}(\zeta)-\sum_{p-0}^{n-k} f^{(k+p)}(z) \frac{(\zeta-z)^{p}}{p !}\right|<\infty
$$

for some $q \in \mathbb{N}$. If the domain $D$ is a quasidisk, i.e., there exists $\delta>0$ such that any two points $\zeta, z \in D$ can be connected by a curve of length at most $\delta|z-\zeta|$, then the Carleman class $A(\bar{D}, \mathcal{M})$ coincides with the class of functions $f$ that are holomorphic in $D$ and, for some $q_{f} \in \mathbb{N}$ and $C_{f}>0$, satisfy $\left|f^{(k)}(z)\right| \leq C_{f} q_{f}^{k} M_{k}, k=0,1,2, \ldots, z \in D$. Obviously, the convex domains enjoy this condition. The problem is as follows: if a point $z_{0}$ is on the boundary (in the plane sense) of $E$, then what conditions on $E$ and the sequence $\mathcal{M}$ ensure that the uniqueness theorem at the point $z_{0}$ will hold true for the class $A(E, \mathcal{M})$ ? The classes where there is no nonzero function vanishing at the point $z_{0}$ together with all its derivatives are said to be quasianalytic at $z_{0}$.

The problem of finding conditions on the sequence $\mathcal{M}$ that are necessary and sufficient for quasianalyticity dates back to Hadamard who posed it in 1912 (see [2]).

Let $I$ be an open interval in $\mathbb{R}$, and let

$$
A(I, \mathcal{M})=\left\{f \in C^{\infty}(I): \sup _{x \in I}\left|f^{(n)}(x)\right| \leq C_{f} q_{f}^{n} M_{n} \text { for all } n \geq 0\right\} .
$$

The class $A(I, \mathcal{M})$ is said to be quasianalytic at a point $x_{0} \in I$ if

$$
f \in A(I, \mathcal{M}), f^{(n)}\left(x_{0}\right)=0 \text { for all } n \geq 0 \Longrightarrow f(x) \equiv 0 .
$$

A criterion for quasianalyticity is given by the Denjoy-Carleman-Ostrowski theorem [3] $[5]$.

Let $T(r)=\sup _{n \geq 0} \frac{r^{n}}{M_{n}}$ be the trace function for the sequence $\mathcal{M}$. The class $A(I, \mathcal{M})$ is quasianalytic at a point $x_{0} \in I$ if and only if

$$
\int_{1}^{\infty} \frac{\ln T(r)}{r^{2}} d r=\infty
$$

As we see, this criterion does not depend on the point $x_{0} \in I$.

A criterion for quasianalyticity at the point $z=0$ for the class $A\left(\bar{\Delta}_{\gamma}, \mathcal{M}\right)$, where

$$
\Delta_{\gamma}=\left\{z:|\arg z|<\frac{\pi}{2} \gamma, 0<|z|<\infty\right\}
$$

is the angle of opening $\gamma \pi$ with vertex at zero, was obtained by Salinas in [6]: for the quasianalyticity of $A\left(\bar{\Delta}_{\gamma}, \mathcal{M}\right)$ at zero it is necessary and sufficient that

$$
\int_{1}^{\infty} \frac{\ln T(r)}{r^{\frac{\gamma+2}{\gamma+1}}} d r=\infty
$$

For a boundary point of the disk, a quasianalyticity condition is given by a theorem due to Korenblum [7]: in this case the condition also does not depend on the point, and the class in question is quasianalytic if and only if

$$
\int_{1}^{\infty} \frac{\ln T(r)}{r^{\frac{3}{2}}} d r=\infty
$$

The quasianalyticity problem for a boundary point $z_{0}$ of a convex bounded domain $D$ was treated in [8] (see also [9]). 
Consider the support lines to the convex domain $D$ through the points at distance from $z_{0}$ equal to the length of an arc $s$, and let $\gamma(s) \pi$ be the size of the angle between these lines that contains the domain $D$. We put

$$
R(x)=\exp ^{\int_{x}^{x_{0}} \frac{1+\gamma(s)}{\gamma(s)} d \ln s}, \quad x \in\left(0 ; x_{0}\right),
$$

where $x_{0}$ is any positive number less than the length of the boundary of $D$. Then the quasianalyticity property is equivalent to the condition

$$
\int_{1}^{\infty} \frac{\ln T(r)}{r^{2} R^{-1}(r)} d r=\infty
$$

where $R^{-1}(r)$ is the function inverse to $R(x)$.

In the present paper, we deal with the quasianalyticity problem at a boundary point $z_{0}$ of a nonconvex domain $D$. We shall pass to the dual problem, which is the problem of density for the system $\left\{\left(\zeta-z_{0}\right)^{(-n)}\right\}, n=1,2, \ldots$, in a certain weighted space of functions holomorphic in the complement of the domain $\bar{D}$. The corresponding spaces can be defined as follows.

In the space $A_{q}(\bar{D}, \mathcal{M})$, we introduce the norm

$$
\|f\|_{q}:=\max \left(\sup _{n \geq 0, k \leq n} \frac{(n-k+1) !}{\sup ^{n+1} M_{n+1}} \frac{\left|R_{n, k}(\zeta, z)\right|}{|\zeta-\zeta|^{n-k+1}}, \frac{1}{M_{0}} \sup _{z \in D}|f(z)|\right) .
$$

The spaces $A_{q}(\bar{D}, \mathcal{M})$ are Banach, and obviously the space $A_{q}(\bar{D}, \mathcal{M})$ is continuously embedded into $A_{q+1}(\bar{D}, \mathcal{M})$. We consider the space $A(\bar{D}, \mathcal{M})$ with the inductive limit topology induced by the spaces $A_{q}(\bar{D}, \mathcal{M})$ :

$$
A(\bar{D}, \mathcal{M})=\operatorname{ind} \lim _{q} A_{q}(\bar{D}, \mathcal{M})
$$

The sequence

$$
m_{n}=\frac{M_{n}}{n !}, \quad n=0,1, \ldots,
$$

is called the adjoint sequence. In what follows, we always assume that the sequence $\left(m_{n}\right)$ is regular [1, i.e., it satisfies the following three conditions:

1) logarithmic convexity:

$$
m_{n}^{2} \leq m_{n-1} m_{n+1}, \quad n=1,2, \ldots ;
$$

2) there is an integer $Q>0$ such that

$$
m_{n+1} \leq Q^{n} m_{n}, \quad n=0,1, \ldots ;
$$

3) the following relation holds true:

$$
\lim _{n \rightarrow \infty} m_{n}^{\frac{1}{n}}=\infty .
$$

We define a function on the positive semiaxis by the formula $M(x)=\sup _{k \geq 0} \frac{1}{m_{k} x^{k}}, x>0$. Clearly, $M(x)$ is a monotone decreasing function and

$$
\lim _{x \rightarrow 0} M(x)=\infty, \quad M(x) \geq \frac{1}{m_{0}} .
$$

The logarithmic convexity of the sequence $\left(m_{n}\right)$ shows that we have an inverse representation:

$$
m_{k}=\sup _{x>0} \frac{1}{x^{k} M(x)}, \quad k=0,1, \ldots
$$


Let $G$ denote the complement of $\bar{D}$ in the extended complex plane, i.e., $G=\overline{\mathbb{C}} \backslash \bar{D}$, and let

$$
d(\zeta)=\inf _{z \in D}|\zeta-z|, \quad \zeta \in G
$$

be the distance function to the boundary of $G$. For $q \in \mathbb{N}$, we introduce the Banach space

$$
X_{q}=\left\{\gamma \in H(G), \gamma(\infty)=0,\|\gamma\|_{X_{q}}=\sup _{\zeta \in G} \frac{|\gamma(\zeta)|}{M(q d(\zeta))}<\infty\right\} .
$$

Since the function $M(x)$ is monotone decreasing, the space $X_{q+1}$ is continuously embedded into $X_{q}$. We denote by $\widetilde{A}(G, \mathcal{M})$ the projective limit of the spaces $X_{q}$ :

$$
\widetilde{A}(G, \mathcal{M})=\underset{q}{\operatorname{proj}} \lim X_{q} .
$$

To simplify the notation, we assume that the point $z=0$ lies on the boundary of $D$ and consider the problem of quasianalyticity at the point $z=0$.

$$
\text { §1. Isomorphism Between the SPACES } A(\bar{D}, \mathcal{M}) \text { AND } \widetilde{A}^{*}(G, \mathcal{M})
$$

Let $\widetilde{A}^{*}(G, \mathcal{M})$ denote the space of continuous linear functionals on $\widetilde{A}(G, \mathcal{M})$ equipped with the strong topology. It is known (see [10]) that

$$
\widetilde{A}^{*}(G, \mathcal{M})=\operatorname{ind} \lim _{q} X_{q}^{*}
$$

Since the function $M(x)$ is bounded from below, the function $(\zeta-z)^{-1}$ belongs to $\widetilde{A}(G, \mathcal{M})$ for any $z \in \bar{D}$. Hence, for every continuous linear functional $S$ on $\widetilde{A}(G, \mathcal{M})$ we can define its Cauchy transform:

$$
\widetilde{S}(z):=S_{\zeta}\left(\frac{1}{\zeta-z}\right), \quad z \in \bar{D} .
$$

Lemma 1. For any $z_{0}, z \in \bar{D}$ and any $k \geq 1$ and $q>0$, we have

$$
\left\|\frac{1}{(\zeta-z)^{k}}-\frac{1}{\left(\zeta-z_{0}\right)^{k}}\right\|_{X_{q}} \leq q^{k+1} m_{k+1} k\left|z-z_{0}\right|
$$

and

$$
\left|\frac{1}{\left(z-z_{0}\right)}\left(\frac{1}{\zeta-z}-\frac{1}{\zeta-z_{0}}\right)-\frac{1}{\left(\zeta-z_{0}\right)^{2}}\right|_{X_{q}} \leq q^{2} m_{2}\left|z-z_{0}\right|
$$

Proof. For $\zeta \in G$, we have

$$
\left|\frac{1}{(\zeta-z)^{k}}-\frac{1}{\left(\zeta-z_{0}\right)^{k}}\right| \leq\left|z-z_{0}\right| \sum_{j=1}^{k} \frac{1}{|\zeta-z|^{j}\left|\zeta-z_{0}\right|^{k-j+1}} \leq \frac{k\left|z-z_{0}\right|}{d(\zeta)^{k+1}} .
$$

Therefore,

$$
\begin{aligned}
\left|\frac{1}{(\zeta-z)^{k}}-\frac{1}{\left(\zeta-z_{0}\right)^{k}}\right| & \leq \frac{k\left|z-z_{0}\right|}{d(\zeta)^{k+1}}=\frac{q^{k+1} m_{k+1} k\left|z-z_{0}\right|}{m_{k+1}(q d(\zeta))^{k+1}} \\
& \leq q^{k+1} m_{k+1} k\left|z-z_{0}\right| M(q d(\zeta)) .
\end{aligned}
$$

This yields the first inequality. The proof of the second is similar.

The second statement of the lemma shows that the function $\widetilde{S}(z)$ is holomorphic in $D$, and moreover,

$$
\widetilde{S}^{\prime}(z)=S_{\zeta}\left(\frac{1}{(\zeta-z)^{2}}\right)
$$


In the same way we obtain a general formula for an arbitrary $k \geq 1$ :

$$
\widetilde{S}^{(k)}(z)=S_{\zeta}\left(\frac{k !}{(\zeta-z)^{k+1}}\right)
$$

Also, the first inequality in Lemma 1 implies that the limit

$$
\widetilde{S}^{(k)}\left(z_{0}\right):=\lim _{z \in D, z \rightarrow z_{0}} \widetilde{S}^{(k)}(z)=S_{z}\left(\frac{k !}{\left(z-z_{0}\right)^{k+1}}\right)
$$

exists for any $z_{0} \in \partial D$, i.e., the function $\widetilde{S}^{(k)}(z)$ has a continuous extension to $\bar{D}$.

Theorem 1. Let $\left(m_{n}\right)$ be a regular sequence, and let $D$ be a Jordan domain. Then the mapping $C: S \longmapsto \widetilde{S}$ is a topological isomorphism between the spaces $\widetilde{A}^{*}(G, \mathcal{M})$ and $A(\bar{D}, \mathcal{M})$.

Proof. First, we verify that $C$ is a continuous mapping from $\widetilde{A}^{*}(G, \mathcal{M})$ to $A(\bar{D}, \mathcal{M})$.

Lemma 2. For any continuous linear functional $S$ on $\widetilde{A}(G, \mathcal{M})$, its Cauchy transform $\widetilde{S}(z)$ is in the space $A(\bar{D}, \mathcal{M})$, and moreover, for any $S \in X_{q}^{*}, q \in \mathbb{N}$, we have

$$
\|\widetilde{S}\|_{A_{q Q}(\bar{D}, \mathcal{M})} \leq q\|S\|_{X_{q}^{*}}
$$

where $Q$ is the number occurring in (1.2).

Proof. For any $\zeta, z, w \in \mathbb{C}, w \neq \zeta, z$, and any $j=0,1,2, \ldots$, the identity

$$
\frac{1}{w-\zeta}-\sum_{p=0}^{j} \frac{(\zeta-z)^{p}}{(w-z)^{p+1}} \equiv \frac{(\zeta-z)^{j+1}}{(w-\zeta)(w-z)^{j+1}}
$$

follows by a straightforward computation of the sum of the geometric progression on the left-hand side.

Differentiating this identity with respect to $w$, we obtain identities valid for $k=0,1$, $2, \ldots$ :

$$
\frac{k !}{(w-\zeta)^{k+1}}-\sum_{p=0}^{j} \frac{(p+k) !(\zeta-z)^{p}}{p !(w-z)^{p+k+1}} \equiv(\zeta-z)^{j+1} \sum_{s=0}^{k} \frac{\left(\begin{array}{l}
k \\
s
\end{array}\right)(j+k-s) ! s !}{j !(w-\zeta)^{s+1}(w-z)^{j+k-s+1}} .
$$

We take a number $n \geq k$ and put $j=n-k$ in the last identity:

$$
\begin{aligned}
\frac{k !}{(w-\zeta)^{k+1}} & -\sum_{p=0}^{n-k} \frac{(p+k) !(\zeta-z)^{p}}{p !(w-z)^{p+k+1}} \\
& \equiv(\zeta-z)^{n-k+1} \sum_{s=0}^{k} \frac{\left(\begin{array}{l}
k \\
s
\end{array}\right)(n-s) ! s !}{(n-k) !(w-\zeta)^{s+1}(w-z)^{n-s+1}} .
\end{aligned}
$$

Now, let $S$ be a continuous linear functional on the space $\widetilde{A}(G, \mathcal{M})$. As has already been mentioned, the Cauchy transform $\widetilde{S}$ extends continuously to the closure of $D$ together with all its derivatives, and formula (1.6) holds true. Therefore, for any $n=0,1,2, \ldots, k \leq n$, by the linearity of the functional we have

$$
\begin{aligned}
\widetilde{S}^{(k)}(\zeta) & -\sum_{p=0}^{n-k} \widetilde{S}^{k+p}(z) \frac{(\zeta-z)^{p}}{p !} \\
& =\widetilde{S}_{w}\left(\frac{k !}{(w-\zeta)^{k+1}}-\sum_{p=0}^{n-k} \frac{(p+k) !}{(w-z)^{p+k+1}} \frac{(\zeta-z)^{p}}{p !}\right), \quad \zeta, z \in \bar{D} .
\end{aligned}
$$


Identity (1.7) implies that

$$
\begin{aligned}
\widetilde{S}^{(k)}(\zeta) & -\sum_{p=0}^{n-k} \widetilde{S}^{k+p}(z) \frac{(\zeta-z)^{p}}{p !} \\
& =(\zeta-z)^{n-k+1} \widetilde{S}_{w}\left(\sum_{s=0}^{k} \frac{\left(\begin{array}{c}
k \\
s
\end{array}\right)(n-s) ! s !}{(n-k) !(w-\zeta)^{s+1}(w-z)^{n-s+1}}\right) .
\end{aligned}
$$

We show that the argument of the function $\widetilde{S}$ on the right-hand side belongs to the space $X_{q}$ for any $q \in \mathbb{N}$.

If $\zeta, z \in \bar{D}$ and $w \notin \bar{D}$, then $|w-\zeta|,|w-z| \geq d(w)$; therefore,

$$
\begin{aligned}
& \left|\sum_{s=0}^{k} \frac{\left(\begin{array}{l}
k \\
s
\end{array}\right)(n-s) ! s !}{(n-k) !(w-\zeta)^{s+1}(w-z)^{n-s+1}}\right| \\
& \quad \leq \frac{1}{d(w)^{n+2}} \sum_{s=0}^{k} \frac{\left(\begin{array}{l}
k \\
s
\end{array}\right)(n-s) ! s !}{(n-k) !}=\frac{k !}{d(w)^{n+2}} \sum_{s=0}^{k}\left(\begin{array}{l}
n-s \\
k-s
\end{array}\right) .
\end{aligned}
$$

The sum of the binomial coefficients on the right-hand side can be evaluated by using the well-known recurrence relation $\left(\begin{array}{c}n+1 \\ m\end{array}\right)=\left(\begin{array}{c}n \\ m\end{array}\right)+\left(\begin{array}{c}n \\ m-1\end{array}\right), m=1,2, \ldots, n$, and $\left(\begin{array}{l}n \\ 0\end{array}\right)=\left(\begin{array}{l}n \\ n\end{array}\right)=1$ :

$$
\begin{aligned}
\sum_{s=0}^{k}\left(\begin{array}{l}
n-s \\
k-s
\end{array}\right) & =\sum_{p=0}^{k}\left(\begin{array}{c}
n-k+p \\
p
\end{array}\right) \\
& =\left(\begin{array}{c}
n-k \\
0
\end{array}\right)+\left(\begin{array}{c}
n-k+1 \\
1
\end{array}\right)+\sum_{p=2}^{k}\left(\begin{array}{c}
n-k+p \\
p
\end{array}\right) \\
& =\left(\begin{array}{c}
n-k+1 \\
0
\end{array}\right)+\left(\begin{array}{c}
n-k+1 \\
1
\end{array}\right)+\sum_{p=2}^{k}\left(\begin{array}{c}
n-k+p \\
p
\end{array}\right) \\
& =\left(\begin{array}{c}
n-k+2 \\
1
\end{array}\right)+\left(\begin{array}{c}
n-k+2 \\
2
\end{array}\right)+\sum_{p=3}^{k}\left(\begin{array}{c}
n-k+p \\
p
\end{array}\right) \\
& =\cdots=\left(\begin{array}{c}
n+1 \\
k
\end{array}\right) .
\end{aligned}
$$

Thus, we obtain the estimate

$$
\begin{aligned}
& \left|\sum_{s=0}^{k} \frac{\left(\begin{array}{c}
k \\
s
\end{array}\right)(n-s) ! s !}{(n-k) !(w-\zeta)^{s+1}(w-z)^{n-s+1}}\right| \\
& \leq \frac{k !}{d(w)^{n+2}}\left(\begin{array}{c}
n+1 \\
k
\end{array}\right)=\frac{1}{d(w)^{n+2}} \frac{(n+1) !}{(n-k+1) !} .
\end{aligned}
$$

Next, for an arbitrary $q \in \mathbb{N}$ and for the number $Q$ occurring in the regularity condition (1.2), by the definition of $M(x)$ we obtain

$$
\begin{aligned}
& \left|\sum_{s=0}^{k} \frac{\left(\begin{array}{l}
k \\
s
\end{array}\right)(n-s) ! s !}{(n-k) !(w-\zeta)^{s+1}(w-z)^{n-s+1}}\right| \leq \frac{1}{m_{n+2}(q d(w))^{n+2}} \frac{q^{n+2} m_{n+2}(n+1) !}{(n-k+1) !} \\
& \quad \leq q M(q d(w))(q Q)^{n+1} \frac{m_{n+1}(n+1) !}{(n-k+1) !}=q M(q d(w))(q Q)^{n+1} \frac{M_{n+1}}{(n-k+1) !} .
\end{aligned}
$$


Obviously, this implies that, for any $\zeta, z \in \bar{D}$,

$$
\sup _{w \in G} \frac{1}{M(q d(w))}\left|\sum_{s=0}^{k} \frac{\left(\begin{array}{l}
k \\
s
\end{array}\right)(n-s) ! s !}{(n-k) !(w-\zeta)^{s+1}(w-z)^{n-s+1}}\right| \leq q \frac{(q Q)^{n+1} M_{n+1}}{(n-k+1) !} .
$$

Since the expression on the left-hand side coincides with the norm in the space $X_{q}$, we have the following estimate for any $\zeta, z \in \bar{D}$ and $q \in \mathbb{N}$ :

$$
\left\|\sum_{s=0}^{k} \frac{\left(\begin{array}{l}
k \\
s
\end{array}\right)(n-s) ! s !}{(n-k) !(w-\zeta)^{s+1}(w-z)^{n-s+1}}\right\|_{X_{q}} \leq q \frac{(q Q)^{n+1} M_{n+1}}{(n-k+1) !} .
$$

We return to identity (1.8). Since we consider the space $\widetilde{A}(G, \mathcal{M})$ with the topology of the canonical projective limit, the functional $S$ on this space can be extended to a continuous linear functional on $X_{q}$ for some $q$. For this number $q$, relation (1.9) implies

$$
\begin{aligned}
\mid \widetilde{S}^{(k)}(\zeta)- & \sum_{p=0}^{n-k} \widetilde{S}^{k+p}(z) \frac{(\zeta-z)^{p}}{p !} \mid \\
& \leq|\zeta-z|^{n-k+1}\left\|S_{w}\right\|_{X_{q}^{*}}\left\|\sum_{s=0}^{k} \frac{\left(\begin{array}{c}
k \\
s
\end{array}\right)(n-s) ! s !}{(n-k) !(w-\zeta)^{s+1}(w-z)^{n-s+1}}\right\|_{X_{q}} .
\end{aligned}
$$

Using this and (1.12), we see that the following estimate is true for all $\zeta, z \in \bar{D}$ and $n \geq 0, k \leq n$ :

$$
\left|\widetilde{S}^{(k)}(\zeta)-\sum_{p=0}^{n-k} \widetilde{S}^{k+p}(z) \frac{(\zeta-z)^{p}}{p !}\right| \leq q|\zeta-z|^{n-k+1}\left\|S_{w}\right\|_{X_{q}^{*}} \frac{(q Q)^{n+1} M_{n+1}}{(n-k+1) !},
$$

or

$$
\sup _{n \geq 0, k \leq n} \frac{(n-k+1) !}{(q Q)^{n+1} M_{n+1}} \sup _{\zeta, z \in \bar{D}} \frac{\left|\widetilde{S}^{(k)}(\zeta)-\sum_{p=0}^{n-k} \widetilde{S}^{k+p}(z) \frac{(\zeta-z)^{p}}{p !}\right|}{|\zeta-z|^{n-k+1}} \leq q\left\|S_{w}\right\|_{X_{q}^{*}} .
$$

Finally, we estimate the supremum of the function $\widetilde{S}(z)$, where $S$ is a functional that admits extension to the space $X_{q}$ :

$$
|\widetilde{S}(z)|=\left|S\left(\frac{1}{w-z}\right)\right| \leq\|S\|_{X_{q}^{*}}\left\|\frac{1}{w-z}\right\|_{X_{q}} .
$$

Since $|w-z| \geq d(w)$ for $w \notin \bar{D}, z \in \bar{D}$, it follows that

$$
\left|\frac{1}{w-z}\right| \leq \frac{1}{d(w)}=\frac{q m_{1}}{q d(w) m_{1}} \leq q m_{1} M(q d(w)) .
$$

Hence,

$$
\left\|\frac{1}{w-z}\right\|_{X_{q}} \leq q m_{1} \leq q Q m_{0}=q M_{0} .
$$

Substituting this estimate in (1.14), we obtain the inequality

$$
\frac{1}{M_{0}} \sup _{z \in \bar{D}}|\widetilde{S}(z)| \leq q\|S\|_{X_{q}^{*}} .
$$

This and estimate (1.13) imply that if a functional $S$ extends to a bounded linear functional on $X_{q}$, then, by the definition of the space $A_{Q q}(\bar{D}, \mathcal{M})$, its Cauchy transform belongs to $A_{Q q}(\bar{D}, \mathcal{M})$, and moreover,

$$
\|\widetilde{S}(z)\|_{A_{q Q}} \leq q\|S\|_{X_{q}^{*}} .
$$

Lemma 2 is proved. 
Our next step is to show that the mapping $C$ is injective. By the Banach theorem, its injectivity will follow from the completeness of the system $\left\{(\zeta-z)^{-1}, z \in D\right\}$ in the space $\widetilde{A}(G, \mathcal{M})$. Since, by Lemma 1 , for any $z \in \partial D$ the function $\frac{1}{\zeta-z}$ can be approximated in $\widetilde{A}(G, \mathcal{M})$ by functions of the form $\left\{(\zeta-z)^{-1}, z \in D\right\}$, it suffices to prove the completeness of the system $\left\{(\zeta-z)^{-1}, z \in \bar{D}\right\}$.

If a function $\gamma(\zeta)$ is holomorphic in $\bar{G}$, we can take a contour $\Gamma$ contained in the domain of analyticity of $\gamma$ and in the domain $D$. We represent the function $\gamma$ in $G$ as the Cauchy integral over $\Gamma$. The integral sums converge to $\gamma$ uniformly in $\bar{G}$. Since the function $M(x)$ is bounded from below, these sums, which are linear combinations of functions belonging to the system under consideration, will approximate $\gamma$ also in the topology of the space $\widetilde{A}(G, \mathcal{M})$. Thus, it remains to prove that the space $H(\bar{G})$ of functions holomorphic in $\bar{G}$ is dense in $\widetilde{A}(G, \mathcal{M})$.

Lemma 3. The space $H(\bar{G})$ is dense in $\widetilde{A}(G, \mathcal{M})$.

Proof. The proof is based on the following theorem by N. Sibony [1].

Theorem A. Let $\Phi$ be a positive function on a domain of holomorphy $\Omega$. Assume that

$$
\Phi(z)-2 \ln \delta_{\Omega}(z)=\left(\sup _{i \in I} \varphi_{i}\right)^{*}(z), \quad z \in \Omega,
$$

where each $\varphi_{i}$ is a plurisubharmonic function in a domain of holomorphy $\Omega_{i} \supset \Omega$. Assume also that the family of restrictions to $\Omega$ of the functions $\varphi_{i}, i \in I$, is right-directed (i.e., for any $i, j \in I$ there exists $k \in I$ such that $\varphi_{i}(z), \varphi_{j}(z) \leq \varphi_{k}(z)$ for all $z \in \Omega$ ). Then for any function $f \in H^{2}(\Omega, \exp (-\Phi))$ there exists a sequence of functions in

$$
\bigcup_{i \in I} H^{2}\left(\Omega_{i}, \exp \left(-\varphi_{i}\right) \delta_{0}^{4}\right)
$$

that converges to $f$ in the norm of the space $H^{2}\left(\Omega, \exp (-\Phi) \delta_{\Omega}^{2} \delta_{0}^{4}\right)$.

Here $\Omega$ is a domain in $\mathbb{C}^{n}, d(z)$ stands for the usual distance to the boundary of $\Omega$, $\delta_{0}(z)=\left(1+|z|^{2}\right)^{-1 / 2}$, and $\delta_{\Omega}=\min \left(d, \delta_{0}\right)$. We denote by $H^{2}(\Omega, w)$ the space of functions holomorphic in $\Omega$ and such that $\int_{\Omega}|f(z)|^{2} w(z) d v(z)<\infty$, where $d v$ is the area Lebesgue measure. The symbol $u^{*}(z)$ denotes the upper regularization of the function $u$ :

$$
u^{*}(z)=\varlimsup_{w \longrightarrow z} u(w) .
$$

Note that in the plane, any domain is a domain of holomorphy.

We choose an exhausting sequence of compact sets $K_{i}$ for the domain $D$ :

$$
K_{i} \subset K_{i+1}, \quad \bigcup_{i=1}^{\infty} K_{i}=D .
$$

For $K_{i}$ we can take $K_{i}=\left\{z \in D: \inf _{w \in \partial D}|w-z| \geq \frac{1}{i}\right\}$.

Let $\Omega_{i}$ denote the set $\mathbb{C} \backslash K_{i}$; the distance to the boundary of $\Omega_{i}$ will be denoted by $d_{i}(\zeta)$, for brevity. Put $\Omega=\mathbb{C} \backslash \bar{D}=G \backslash\{\infty\}$. We fix $q \in \mathbb{N}$ and put $q_{1}=2 Q q$, where $Q$ is as in (1.2). Let

$$
\begin{aligned}
\varphi_{i}(\zeta) & =2 \ln M\left(q_{1} d_{i}(\zeta)\right), \quad \zeta \in \Omega_{i}, \\
\Phi(\zeta) & =2 \ln M\left(q_{1} d(\zeta)\right)+2 \ln \delta_{\Omega}(\zeta), \quad \zeta \in \Omega .
\end{aligned}
$$

By definition, $\ln M(q x)$ is a convex and monotone increasing function of $-\ln x$, and the function $-\ln d(\zeta)$ is subharmonic in $\Omega_{i}$. Therefore, the functions $\varphi_{i}$ are subharmonic in 
$\Omega_{i}$. Since, for any $\zeta \in \Omega$, the sequence $d_{i}(\zeta)$ is monotone nonincreasing and tends to $d(\zeta)$, and the function $M(x)$ is monotone nonincreasing, we have

$$
\Phi(\zeta)-2 \ln \delta_{\Omega}(\zeta)=\sup _{i} \varphi_{i}(\zeta), \quad \zeta \in \Omega
$$

In what follows we shall need a property of the weight function $M(x)$.

Lemma 4. For any $x>0$, we have

$$
M(Q x) \leq x M(x),
$$

where $Q$ is the number occurring (1.2).

Proof. This follows immediately from the definition of $M(x)$ and condition (1.2):

$$
M(Q x)=\sup _{k \geq 0} \frac{1}{m_{k} x^{k} Q^{k}} \leq \sup _{k \geq 0} \frac{1}{m_{k+1} x^{k}} \leq x M(x) .
$$

Theorem A deals with integral norms, whereas the norms in the spaces under consideration are uniform. Therefore, we need yet another lemma to translate the results from integral to uniform norms.

Lemma 5. If $f \in H^{2}\left(\Omega, \exp (-\Phi) \delta_{\Omega}^{2} \delta_{0}^{4}\right)$, then

$$
|f(\zeta)| \leq \frac{2 q \sqrt{6}}{\sqrt{\pi}} M(q d(\zeta))\left(1+|\zeta|^{2}\right)\|f\|, \quad \zeta \in \Omega,
$$

where $\|f\|$ denotes the norm in the space $H^{2}\left(\Omega, \exp (-\Phi) \delta_{\Omega}^{2} \delta_{0}^{4}\right)$.

Proof. By the subharmonicity of $|f|^{2}$, we have

$$
\begin{aligned}
|f(\zeta)|^{2} & \leq \frac{4}{\pi d(\zeta)^{2}} \int_{|\lambda-\zeta| \leq d(\zeta) / 2}|f(\lambda)|^{2} d v(\lambda) \\
& \leq \frac{4}{\pi d(\zeta)^{2}} \max _{|\lambda-\zeta| \leq d(\zeta) / 2}\left(e^{\Phi(\lambda)} \delta_{\Omega}^{-2}(\lambda) \delta_{0}^{-4}(\lambda)\right)\|f\| .
\end{aligned}
$$

Obviously, in the disk of integration we have $d(\lambda) \geq d(\zeta) / 2$; therefore,

$$
M\left(q_{1} d(\lambda)\right) \leq M\left(\frac{q_{1}}{2} d(\zeta)\right) .
$$

The estimate $d(z) \leq|z|$ shows that $|\lambda| \leq \frac{3}{2}|\zeta|$ for the points of the disk of integration. Hence, in that disk, $\left(1+|\lambda|^{2}\right)^{2} \leq \frac{81}{16}\left(1+|\zeta|^{2}\right)^{2}$. Also, since $q_{1}=2 Q q$, we have (Lemma 4)

$$
M\left(q_{1} d(\lambda)\right) \leq q d(\zeta) M(q d(\zeta)) .
$$

Thus,

$$
\max _{|\lambda-\zeta| \leq d(\zeta) / 2}\left(e^{\Phi(\lambda)} \delta_{\Omega}^{-2}(\lambda) \delta_{0}^{-4}(\lambda)\right) \leq 6 q^{2} d(\zeta)^{2} M^{2}(q d(\zeta))\left(1+|\zeta|^{2}\right)^{2}
$$

Substituting this in (1.15), we obtain the required estimate.

Lemma 5 is proved.

Now, we take an arbitrary function $\gamma \in \widetilde{A}(G, \mathcal{M})$. By Lemma 1 , the function $\zeta^{-k}$ can be approximated by functions in $H(\bar{G})$, and so we can omit any finite number of terms in the Laurent series for $\gamma$ at $\infty$. We omit the first two terms and assume that

$$
|\gamma(\zeta)|^{2}=O\left(\frac{1}{|\zeta|^{6}}\right), \quad|\zeta| \rightarrow \infty .
$$

The definition of the space $\widetilde{A}(G, \mathcal{M})$ implies the inequality

$$
|\gamma(\zeta)| \leq C M\left(q_{1} Q d(\zeta)\right), \quad \zeta \in \Omega .
$$


Hence, by Lemma 4, we obtain $|\gamma(\zeta)| \leq C q_{1} d(\zeta) M\left(q_{1} d(\zeta)\right), \zeta \in \Omega$; together with (1.16), this yields

$$
|\gamma(\zeta)|^{2} e^{-\Phi(\zeta)} \leq \frac{\text { const }}{(1+|\zeta|)^{4}}, \quad \zeta \in \Omega
$$

that is, $\gamma \in H^{2}(\Omega, \exp (-\Phi))$. We apply Theorem A to this function: the function $\gamma$ can be approximated by functions belonging to

$$
\bigcup_{i} H^{2}\left(\Omega_{i}, e^{-\varphi_{i}} \delta_{0}^{4}\right)
$$

in the norm of the space $H^{2}\left(\Omega \exp (-\Phi) \delta_{\Omega}^{2} \delta_{0}^{4}\right)$. Unfortunately, the approximating functions may fail to be holomorphic at the point $z=\infty$, and so we need to correct them slightly. Let $f_{n}$ be the approximating sequence and let $g_{n}=f_{n}-\gamma$; then, by Lemma 5 , we have

$$
\left|g_{n}(\zeta)\right| \leq \epsilon_{n} M(q d(\zeta))\left(1+|\zeta|^{2}\right), \quad \zeta \in \Omega,
$$

where $\epsilon_{n} \rightarrow 0$. The function $M(x)$ is bounded from above for $x \geq 1$. Hence, the regular part of the Laurent expansion for $g_{n}$ in a neighborhood of $z=\infty$ contains at most three terms:

$$
g_{n}(\zeta)=P_{n}(\zeta)+\gamma_{n}(\zeta),
$$

where $P_{n}$ is a polynomial of degree at most 2, the functions $\gamma_{n}$ are holomorphic at the point $z=\infty$ and $\gamma_{n}(\infty)=0$. For $k \in \mathbb{N}$, let $\Gamma_{k}$ be the contour $\{\zeta \in \Omega: d(\zeta)=k\}$, and let $R_{k}=\max _{\zeta \in \Gamma_{k}}|\zeta|$. It is clear that $\min \left\{|\zeta-\lambda|, \zeta \in \Gamma_{1}, \lambda \in \Gamma_{2}\right\} \geq 1$. Hence, by (1.17), inside the contour $\Gamma_{1}$ we have

$$
\left|P_{n}(\zeta)\right|=\left|\int_{\Gamma_{2}} \frac{g_{n}(\lambda)}{\lambda-\zeta} d \lambda\right| \leq \epsilon_{n} l_{2}\left(1+R_{2}^{2}\right) M(2 q),
$$

where $l_{2}$ is the length of $\Gamma_{2}$. Since the function $M(x)$ is monotone decreasing and $d(\zeta)<1$ inside the contour $\Gamma_{1}$, it follows that

$$
\left|P_{n}(\zeta)\right| \leq \epsilon_{n} l_{2}\left(1+R_{2}^{2}\right) M(q d(\zeta)) .
$$

Combining this and (1.17), we conclude that in $\Omega$ inside the contour $\Gamma_{1}$ we have

$$
\left|\gamma_{n}(\zeta)\right| \leq \epsilon_{n}\left(l_{2}+1\right)\left(1+R_{2}^{2}\right) M(q d(\zeta)) .
$$

On the contour $\Gamma_{1}$ this estimate takes the form

$$
\left|\gamma_{n}(\zeta)\right| \leq \epsilon_{n}\left(l_{2}+1\right)\left(1+R_{2}^{2}\right) M(q) .
$$

Using the maximum principle for the points outside $\Gamma_{1}$ and the monotonicity of $M(x)$, we obtain

$$
\begin{aligned}
\left|\gamma_{n}(\zeta)\right| & \leq \epsilon_{n}\left(l_{2}+1\right)\left(1+R_{2}^{2}\right) M(q)=\epsilon_{n}\left(l_{2}+1\right)\left(1+R_{2}^{2}\right) M(q) m_{0} \frac{1}{m_{0}} \\
& \leq \epsilon_{n}\left(l_{2}+1\right)\left(1+R_{2}^{2}\right) M(q) m_{0} M(q d(\zeta)) .
\end{aligned}
$$

Combined with (1.18), the latter estimate shows that

$$
\left|\gamma_{n}(\zeta)\right| \leq \epsilon_{n}^{\prime} M(q d(\zeta)), \quad \zeta \in \Omega,
$$

where $\epsilon_{n}^{\prime} \rightarrow 0$. It remains to observe that $\gamma_{n}=g_{n}-P_{n}=\left(f_{n}-P_{n}\right)-\gamma$. Thus, the sequence $f_{n}-P_{n}$ approximates the function $\gamma$ in the space $X_{q}$, and, by construction, these functions are holomorphic in $\bar{\Omega}$, including the point $z=\infty$, i.e., $f_{n}-P_{n} \in H(\bar{G})$.

Lemma 3 is proved. 
To complete the proof of Theorem 1, it remains to prove that the mapping $C$ is surjective.

Let $f \in A(\bar{D}, \mathcal{M})$; we construct a continuous linear functional on $\widetilde{A}(G, \mathcal{M})$ such that $\widetilde{S}=f$. By Lemma 3 , the space $H(\bar{G})$ is dense in $\widetilde{A}(G, \mathcal{M})$. Hence, it suffices to define a continuous linear functional on $H(\bar{G})$ and then extend it by continuity to $\widetilde{A}(G, \mathcal{M})$.

For any function $\gamma \in \widetilde{A}(G, \mathcal{M})$ holomorphic on $\bar{G}$, put

$$
S(\gamma)=\frac{1}{2 \pi i} \int_{\partial D} \gamma(z) f(z) d z .
$$

Note that we can choose a smooth contour $\Gamma_{\gamma}$, contained in the intersection of the domain of holomorphy of the function $\gamma$ with $D$, in such a way that

$$
S(\gamma)=\frac{1}{2 \pi i} \int_{\Gamma_{\gamma}} \gamma(z) f(z) d z
$$

We will need this remark when we apply the Green formula.

We make use of a theorem on pseudoanalytic continuation proved in [1].

Theorem B. Let $D$ be a domain in $\mathbb{C}$, and let $m_{n}=\frac{M_{n}}{n !}$ be a regular sequence. Then any function $f$ in the class $A(\bar{D}, \mathcal{M})$ can be extended to a continuously differentiable function $F$ with compact support in $\mathbb{C}$ such that

$$
\left|\frac{\partial F}{\partial \bar{\zeta}}\right| \leq \frac{C}{M(B d(\zeta))}, \quad \zeta \in \mathbb{C},
$$

where $C$ and $B$ are positive constants.

With the help of this theorem, we extend the function $f \in A(\bar{D}, \mathcal{M})$ to a function $F$ and apply the Green formula

$$
\begin{aligned}
S(\gamma) & =\frac{1}{2 \pi i} \int_{\Gamma_{\gamma}} \gamma(z) f(z) d z=\frac{1}{2 \pi i} \int_{\Gamma_{\gamma}} \gamma(z) F(z) d z \\
& =-\frac{1}{\pi} \int_{G} \gamma(\zeta) \frac{\partial F(\zeta)}{\partial \bar{\zeta}} d v(\zeta)=-\frac{1}{\pi} \int_{K \cap G} \gamma(\zeta) \frac{\partial F(\zeta)}{\partial \bar{\zeta}} d v(\zeta),
\end{aligned}
$$

where $K$ is the support of $F$ and $d v(\zeta)$ is the area Lebesgue measure. This representation implies the estimate

$$
|S(\gamma)| \leq \frac{|K| C}{\pi} \sup _{\zeta \in G} \frac{|\gamma(\zeta)|}{M(B d(\zeta))}=2|K| C\left\|_{\gamma}\right\|_{X_{B}},
$$

where $|K|$ is the area of the compact set $K$ and $\|\gamma\|_{X_{B}}$ is the norm of $\gamma$ in the space $X_{B}$. Thus, $S$ is a linear functional on $H(\bar{G})$ continuous with respect to the norm of $X_{B}$, and hence, also in the topology of the space $\widetilde{A}(G, \mathcal{M})$. By the density of $H(\bar{G})$ in $\widetilde{A}(G, \mathcal{M})$, the functional $S$ can be extended to a continuous linear functional on $\widetilde{A}(G, \mathcal{M})$. By the definition and the Cauchy formula, we conclude that $\widetilde{S}(z)=f(z), z \in D$.

Theorem 1 is proved.

\section{§2. Quasianalyticity and an extremal problem FOR SUBHARMONIC FUNCTIONS}

Theorem 1, Lemma 1, and the Banach theorem imply the following criterion for quasianalyticity.

Theorem 2. Let the sequence $\left(m_{n}\right)$ be regular, and let the point $z=0$ be on the boundary of a bounded Jordan domain $D$. The class $A(\bar{D}, \mathcal{M})$ is quasianalytic at the point $z=0$ if and only if the system $\zeta^{-n}, n=1,2, \ldots$, is dense in the space $\widetilde{A}(G, \mathcal{M})$. 
Proof. If $S$ is a continuous linear functional on $\widetilde{A}(G, \mathcal{M})$ that is equal to zero on the elements of the system $\zeta^{-n}, n=1,2, \ldots$, then, by Lemma 1 , the function $\widetilde{S}(z)$ in $A(\bar{D}, \mathcal{M})$ satisfies the condition $\widetilde{S}^{(n)}(0)=0$ for all $n=1,2, \ldots$

Theorem 2 is proved.

Let $q \in \mathbb{N}$ be arbitrary. For any $w \in G$, there exists a number $p \in \mathbb{N}$ and a point $t \in \partial D$ such that

$$
M(q d(w))=\sup _{k \geq 0} \sup _{z \in D}\left|\frac{1}{q^{k} m_{k}(z-w)^{k}}\right|=\left|\frac{1}{q^{p} m_{p}(t-w)^{p}}\right| .
$$

In what follows we denote the function $\frac{1}{q^{p} m_{p}(t-\zeta)^{p}}$ by $f_{w}(\zeta)$. Thus, the function $f_{w}(\zeta)$ has the following properties:

$$
f_{w}(\zeta) \in \widetilde{A}(G, \mathcal{M}) ; \quad\left|f_{w}(\zeta)\right| \leq M(q d(\zeta)), \quad \zeta \in G ; \quad\left|f_{w}(w)\right|=M(q d(w)) .
$$

Assume that the system $\left\{\zeta^{-n}, n=1,2, \ldots\right\}$ is complete in $\widetilde{A}(G, \mathcal{M})$. This means that for any function $\gamma$ in $\widetilde{A}(G, \mathcal{M})$ there exists a sequence of polynomials $P_{n}(z), P_{n}(0)=0$, $n=0,1, \ldots$, such that

$$
P_{n}\left(\frac{1}{\zeta}\right) \rightarrow \gamma(\zeta)
$$

in the space $\widetilde{A}(G, \mathcal{M})$ as $n \rightarrow \infty$. In particular, for any $\epsilon>0$ there exists a polynomial $P(z), P(0)=0$, such that

$$
\left|P\left(\frac{1}{\zeta}\right)-f_{w}(\zeta)\right| \leq \epsilon M(q d(\zeta)), \quad \zeta \in G
$$

Then

$$
\left|P\left(\frac{1}{\zeta}\right)\right| \leq(1+\epsilon) M(q d(\zeta)), \quad \zeta \in G ; \quad\left|P\left(\frac{1}{w}\right)\right| \geq(1-\epsilon) M(q d(w)) .
$$

For the polynomial $P_{1}(z)=P(z) /(1+\epsilon)$ we have the inequalities

$$
\left|P_{1}\left(\frac{1}{\zeta}\right)\right| \leq M(q d(\zeta)), \quad \zeta \in G ; \quad\left|P_{1}\left(\frac{1}{w}\right)\right| \geq \frac{1-\epsilon}{1+\epsilon} M(q d(w)) .
$$

In view of these considerations, we introduce the class $K_{q}$ of functions $v$ satisfying the following conditions:

1) every function $v$ is continuous and subharmonic in $\overline{\mathbb{C}} \backslash\{0\}$;

2) $v(\zeta)=O\left(\ln \frac{1}{|\zeta|}\right)$ as $\zeta \rightarrow 0$

3) $v(\zeta) \leq \ln M(q d(\zeta)), \quad \zeta \in G$.

For example, the functions $\max \left(\ln \left|P_{1}\left(\frac{1}{\zeta}\right)\right|,-\ln m_{0}\right)$, where the polynomials $P_{1}$ satisfy (2.1), are in $K_{q}$.

Obviously, instead of the sequence $M_{n}$ we may consider the sequence $M_{n} / e M_{0}$ and assume that $m_{0}=1 / e$. Thus, $M(x) \geq 1 / m_{0}=e$ and $\ln M(x) \geq 1$. Therefore, to the definition of the class $K_{q}$ we may add the following item:

4) $v(z) \geq 0$.

Theorem 3. Let the sequence $\left(m_{n}\right)$ be regular, and let the point $z=0$ be on the boundary of a bounded Jordan domain $D$. The class $A(\bar{D}, \mathcal{M})$ is quasianalytic at the point $z=0$ if and only if the condition

$$
\sup \left\{v(\zeta), v \in K_{q}\right\}=\ln M(q d(\zeta)), \quad \zeta \in G,
$$

is fulfilled for each $q \in \mathbb{N}$. 
Proof. By Theorem 2, the quasianalyticity of the class $A(\bar{D}, \mathcal{M})$ implies that the system $\zeta^{-n}, n=1,2, \ldots$, is complete in the space $\widetilde{A}(G, \mathcal{M})$. Above, we have shown that the completeness of this system implies $(2.2)$, because the functions $\max \left(\ln \mid\left(P_{1}\left(\frac{1}{\zeta}\right) \mid,-\ln m_{0}\right)\right.$ belong to the class $K_{q}$. To prove the converse statement, we use the following lemma.

Lemma 6. Assume that the condition

$$
\sup \left\{v(\zeta), v \in K_{q}\right\}=\ln M(q d(\zeta)), \quad \zeta \in G,
$$

is fulfilled for some $q$. Then any function in the space $\widetilde{A}(G, \mathcal{M})$ can be approximated by the system $\zeta^{-n}, n=1,2, \ldots$, in the norm of the space $X_{q / 2 Q}$, where $Q$ is as in (1.2).

Proof. For the role of $\varphi_{i}$ in Theorem A, we take functions $2 v(\zeta)$, where $v \in K_{q}$. Let $\Omega_{i}=\mathbb{C} \backslash\{0\}, \Omega=\mathbb{C} \backslash \bar{D}$. We put

$$
\Phi(\zeta)=2 \ln M(q d(\zeta))+2 \ln \delta_{G}(\zeta), \quad \zeta \in \Omega .
$$

Then the assumptions of Theorem A are satisfied in view of the assumptions of the lemma. Therefore, any function of the class $H^{2}(\Omega, \exp (-\Phi))$ can be approximated by functions belonging to the union

$$
\bigcup_{v \in K_{q}} H^{2}\left(\Omega_{i}, \exp (-2 v(\zeta)) \delta_{0}^{4}\right)
$$

in the norm of the space $H^{2}\left(\Omega, \exp (-\Phi) \delta_{G}^{2} \delta_{0}^{4}\right)$. Let $\gamma \in \widetilde{A}(G, \mathcal{M})$. Since we are interested in approximating the function $\gamma$ by linear combinations of functions $\zeta^{-n}$, we can omit several terms in the Laurent series expansion of $\gamma$ near $\infty$. Thus, we may assume that

$$
|\gamma(\zeta)|^{2}=O\left(\ln \frac{1}{|\zeta|^{6}}\right), \quad|\zeta| \rightarrow \infty
$$

Also, by Lemma 4 we have

$$
|\gamma(\zeta)| \leq\|\gamma\|_{X_{q Q}} M(q Q d(\zeta)) \leq\|\gamma\|_{X_{q Q}} q d(\zeta) M(q d(\zeta)), \quad \zeta \in G .
$$

These two relations show that $\gamma \in H^{2}(\Omega, \exp (-\Phi))$. Let

$$
f_{n} \in \bigcup_{v \in K_{q}} H^{2}\left(\mathbb{C} \backslash\{0\}, \exp (-2 v(\zeta)) \delta_{0}^{4}\right)
$$

be an approximating sequence and let $g_{n}=f_{n}-\gamma$. By Lemma 5 , we have

$$
\left|g_{n}(\zeta)\right| \leq \epsilon_{n} M\left(q_{1} d(\zeta)\right)\left(1+|\zeta|^{2}\right), \quad \zeta \in \Omega,
$$

where $\epsilon_{n} \rightarrow 0$ and $q_{1}=q / 2 Q$. The function $M(x)$ is bounded from above for $x \geq 1$, and so the last estimate yields

$$
g_{n}(\zeta)=Q_{n}(\zeta)+\gamma_{n}(\zeta)
$$

where $Q_{n}$ is a polynomial of degree at most 2 , and $\gamma_{n}(\infty)=0$. Since $\gamma$ is holomorphic at the point $\zeta=\infty$ and $\gamma(\infty)=0$, we conclude that $Q_{n}(\zeta)$ is the regular part of the function $f_{n}$ at $\infty$, and the function $f_{n}-Q_{n}$ is holomorphic at $\infty$ and vanishes there. As in the proof of Theorem 1, we can show that

$$
\left|\gamma_{n}(\zeta)\right| \leq \epsilon_{n}^{\prime} M\left(q_{1} d(\zeta)\right), \quad \zeta \in G,
$$

where $\epsilon_{n}^{\prime} \rightarrow 0$. This means that the functions $f_{n}(\zeta)-Q_{n}(\zeta)$ approximate $\gamma$ in the norm of the space $X_{q_{1}}$. The function $f_{n}$ belongs to one of the spaces

$$
H^{2}\left(\mathbb{C} \backslash\{0\}, \exp (-2 v(\zeta)) \delta_{0}^{4}\right) .
$$

Hence,

$$
\int_{\mathbb{C} \backslash\{0\}}\left|f_{n}(\zeta)\right|^{2} \frac{e^{-2 v(\zeta)}}{\left(1+|\zeta|^{2}\right)^{2}} d v(\zeta)<\infty .
$$


Now the definition of the classes $K_{q}$ and the subharmonicity of $\left|f_{n}\right|$ show that the function $f_{n}$ has a pole of order $N$ at the point $\zeta=0$. Since $f_{n}-Q_{n}$ is holomorphic at infinity, this function is a linear combination of the functions $\zeta^{-n}, n=1,2, \ldots, N$. Thus, we have shown that the function $\gamma$ can be approximated by the system $\zeta^{-n}$ in the norm of the space $X_{q / 2 Q}$.

Lemma 6 is proved.

Now we complete the proof of Theorem 3. If condition (2.2) is satisfied for all $q \in \mathbb{N}$, then, by Lemma 6 , the functions $\widetilde{A}(G, \mathcal{M})$ can be approximated by the system $\left(\zeta^{-n}\right)$ in the norm of each of the spaces $X_{q / 2 Q}$, that is, in the topology of the space $\widetilde{A}(G, \mathcal{M})$.

Theorem 3 is proved.

\section{§3. Quasianalyticity and a Dirichlet PRoblem}

We introduce the function

$$
U_{q}(\zeta)=\sup \left\{v(\zeta), v \in K_{q}\right\}, \quad \zeta \in \overline{\mathbb{C}}
$$

Lemma 7. For any $q \in \mathbb{N}$, either $U_{q}(\zeta) \equiv \infty$ in $D$ or $U_{q}(\zeta)$ is a harmonic function in $D$.

Proof. Let $D_{1}$ denote the set of points $z$ in $D$ such that $U_{q}(z)=\infty$, and let $D_{2}=$ $\left\{z \in D: U_{q}(z)<\infty\right\}$. We fix an arbitrary point $z_{0} \in D$ and a monotone increasing sequence of functions $v_{n} \in K_{q}$ such that $\lim _{n \rightarrow \infty} v_{n}\left(z_{0}\right)=U_{q}\left(z_{0}\right)$. Let $2 d=\inf _{\zeta \in G}\left|\zeta-z_{0}\right|$. We extend each function $v_{n}$ harmonically to the disk $B\left(z_{0}, d\right)$. Obviously, the resulting functions $\widetilde{v}_{n}$ are also in $K_{q}$, and moreover, since $\widetilde{v}_{n} \geq v_{n}$, we have $\lim _{n \rightarrow \infty} \widetilde{v}_{n}\left(z_{0}\right)=$ $U_{q}\left(z_{0}\right)$. Applying the Harnack inequality to each of these functions, we see that, in the disk $B\left(z_{0}, d / 2\right)$, we have

$$
\frac{1}{3} \widetilde{v}_{n}\left(z_{0}\right) \leq \widetilde{v}_{n}(z) \leq 3 \widetilde{v}_{n}\left(z_{0}\right) .
$$

The left-hand side inequalities show that if $z_{0} \in D_{1}$, then $B\left(z_{0}, d / 2\right) \subset D_{1}$, whereas the right-hand side inequalities show that for $z_{0} \in D_{2}$ we have $B\left(z_{0}, d / 2\right) \subset D_{2}$. Thus, the two sets $D_{1}$ and $D_{2}$ are open in $D$. Since $D$ is connected, this means that one of them must be empty.

It remains to show that if $D_{1}=\varnothing$, then the function $U_{q}$ is harmonic in $D$.

We take an arbitrary point $w \in B\left(z_{0}, d / 2\right)$ and, as for the point $z_{0}$, construct a monotone increasing sequence of functions $h_{n}(z) \in K_{q}$ such that $\lim _{n \rightarrow \infty} h_{n}(w)=U_{q}(w)$. Then, by harmonic extension to the disk $B\left(z_{0}, d\right)$, we obtain an increasing sequence of functions $\widetilde{h}_{n}$ with the same property: $\lim _{n \rightarrow \infty} \widetilde{h}_{n}(w)=U_{q}(w)$.

Now we put $s_{n}(z)=\max \left(v_{n}(z), h_{n}(z)\right)$. Clearly,

$$
\lim _{n \rightarrow \infty} s_{n}(w)=U_{q}(w), \quad \lim _{n \rightarrow \infty} s_{n}\left(z_{0}\right)=U_{q}\left(z_{0}\right) .
$$

Extending the $s_{n}$ harmonically to the disk $B\left(z_{0}, d\right)$, we construct functions $\widetilde{s}_{n}$ with the same properties. Also, it is clear that $\widetilde{s}_{n}$ is greater than both $\widetilde{v}_{n}$ and $\widetilde{h}_{n}$. Put

$$
\lim _{n \rightarrow \infty} \widetilde{v}_{n}(z)=V(z), \quad \lim _{n \rightarrow \infty} \widetilde{h}_{n}(z)=H(z), \quad \lim _{n \rightarrow \infty} \widetilde{s}_{n}(z)=S(z) .
$$

Then from our constructions it follows that

$$
\begin{array}{ccc}
V\left(z_{0}\right)=U_{q}\left(z_{0}\right), \quad H(w)=U_{q}(w), \quad S\left(z_{0}\right)=U_{q}\left(z_{0}\right), \quad S(w)=U_{q}(w), \\
S(z) \geq V(z), \quad S(z) \geq H(z) .
\end{array}
$$

A nonnegative function $S-V$ harmonic in $B\left(z_{0}, d\right)$ vanishes at the interior point $z_{0}$. By the maximum principle, $S \equiv V$. Similarly, $S \equiv H$. Therefore, $V(w)=S(w)=$ $H(w)=U_{q}(w)$, but the construction of $V$ depends only on $z_{0}$ and not on $w$. Hence, 
$V(w)=U_{q}(w)$ for all points in the disk $B\left(z_{0}, d / 2\right)$, and $U_{q}(z)$ is harmonic in this disk. Since $z_{0}$ is an arbitrary point in $D$, we conclude that $U_{q}$ is harmonic in $D$.

Lemma 7 is proved.

Lemma 8. If for $q \in \mathbb{N}$ we have $U_{q}(\zeta) \equiv \infty$ in $D$, then $U_{q}(\zeta) \equiv \ln M(q d(\zeta))$ in $G$.

Proof. For a fixed $q \in \mathbb{N}$ and any $w \in G$, in $\S 2$ we introduced the function $f_{w}(\zeta)$ of the form $\left(m_{p} q^{p}(\zeta-z)^{p}\right)^{-1}$, where $p \in \mathbb{N}$ and $z$ is a point on the boundary of $D$. These functions have the properties

$$
\left|f_{w}(\zeta)\right| \leq M(q d(\zeta)), \quad \zeta \in G, \quad\left|f_{w}(w)\right|=M(q d(w)) .
$$

Fixing a point $w \in G$ and a number $\epsilon>0$, we replace the boundary point $z$ in the definition of $f_{w}(\zeta)$ with a sufficiently close point $z^{\prime} \in D$ so that the resulting function $\tilde{f}_{w}(\zeta)$ satisfy

$$
\left|\tilde{f}_{w}(\zeta)\right| \leq M(q d(\zeta)), \quad \zeta \in G ; \quad\left|\tilde{f}_{w}(w)\right| \geq(1-\epsilon) M(q d(w)) .
$$

By the assumptions of the lemma, we have $U_{q}\left(z^{\prime}\right)=\infty$, and so there exists a sequence of functions $v_{n} \in K_{q}$ such that $v_{n}\left(z^{\prime}\right) \rightarrow \infty$. As in the proof of the preceding lemma, we may assume that the functions $v_{n}$ are extended harmonically to the disk $B\left(z^{\prime}, d\right)$, where $2 d$ is the distance from $z^{\prime}$ to the boundary $D$. Then, by (3.1), $v_{n}(z) \rightarrow \infty$ uniformly in the disk $B\left(z^{\prime}, d / 2\right)$. Outside the disk $B\left(z^{\prime}, d / 3\right)$, the function $\left|\widetilde{f}_{w}(\zeta)\right|$ is bounded:

$$
\left|\widetilde{f}_{w}(\zeta)\right| \leq \frac{3^{p}}{m_{p} q^{p} d^{p}}=M .
$$

We choose $n$ so large that $v_{n}(\zeta)>\ln M$ in the disk $B\left(z^{\prime}, d / 2\right)$ and introduce the function

$$
u(\zeta)= \begin{cases}\max \left(v_{n}(\zeta), \ln \left|\widetilde{f}_{n}(\zeta)\right|\right) & \text { if } \zeta \notin B\left(z^{\prime}, d / 3\right) \\ v_{n}(\zeta) & \text { if } \zeta \in B\left(z^{\prime}, d / 3\right)\end{cases}
$$

By construction, we have $u \in K_{q}$ and

$$
u(w) \geq \ln \left|\widetilde{f}_{w}(w)\right| \geq \ln (1-\epsilon)+\ln M(q d(w)) .
$$

Since $\epsilon>0$ is arbitrary, $U_{q}(w)=\ln M(q d(w))$.

Lemma 8 is proved.

Lemma 9. If, for a given $q$, the function $U_{q}(z)$ is finite at some point $z_{1} \in D$, then in a neighborhood of any point $z \in \partial D$ there are points $\zeta \in G$ such that $U_{q}(\zeta)<\ln M(q d(\zeta))$.

Proof. Assume the contrary. Let $z_{0} \in \partial D$ and suppose that $U_{q}(\zeta) \equiv \ln M(q d(\zeta))$ in the intersection of the disk $B\left(z_{0}, r\right)$ with $G$. Put $r_{0}=\min \left(r / 2,\left|z_{0}\right| / 2\right)$ and let $G^{\prime}$ be the connected component of the intersection of $G$ with $B\left(z_{0}, r_{0}\right)$ such that $z_{0}$ is on its boundary. We denote by $G_{0}$ the difference $G \backslash G^{\prime}$ and let $\widetilde{K}_{q}$ be the class of functions that are subharmonic, nonnegative, and continuous in $\overline{\mathbb{C}} \backslash\{0\}$ and satisfy the conditions

$$
v(z)=O\left(\ln \frac{1}{|z|}\right), \quad|z| \rightarrow 0, \quad v(\zeta) \leq \ln M(q d(\zeta)), \quad \zeta \in G_{0}
$$

Obviously, we have $K_{q} \subset \widetilde{K}_{q}$, whence

$$
\widetilde{U}_{q}(\zeta)=\sup \left\{v(\zeta), v \in \widetilde{K}_{q}\right\} \geq U_{q}(\zeta) .
$$

Hence, the function $\widetilde{U}_{q}(\zeta)$ is unbounded near the point $z_{0} \in \mathbb{C} \backslash \bar{G}_{0}$. By Lemma 7, we see that $\widetilde{U}_{q}(\zeta) \equiv \infty$ in $D_{0}=\mathbb{C} \backslash \bar{G}_{0} \supset D$. Let $\widetilde{v}$ be a function in $\widetilde{K}_{q}$ such that

$$
\widetilde{v}\left(z_{1}\right) \geq U_{q}\left(z_{1}\right)+4 \text {. }
$$


Then the function $v(z)=\widetilde{v}(z)-2$ satisfies the inequalities

$$
v\left(z_{1}\right) \geq U_{q}\left(z_{1}\right)+2, \quad v(\zeta) \leq \ln M(q d(\zeta))-2, \quad \zeta \in G_{0} .
$$

The subharmonic function $v(z)$ is bounded from above in the disk $B\left(z_{0}, r_{0}\right)$ :

$$
v(z) \leq M, \quad z \in B\left(z_{0}, r_{0}\right),
$$

for some $M$. Since $M(x)$ is monotone, on the set $G^{\prime \prime}=\left\{\zeta \in G^{\prime}: d(\zeta) \leq \epsilon\right\}$ we have

$$
\ln M(q d(\zeta)) \geq \ln M(q \epsilon) .
$$

Let $\epsilon>0$ be so small that $\ln M(q \epsilon)>M+1$. Then in $G^{\prime \prime}$ we have the inequality

$$
\ln M(q d(\zeta)) \geq M+1 \text {. }
$$

By assumption, the identity $U_{q}(z) \equiv \ln M(q d(z))$ is true on the set $G^{\prime} \backslash G^{\prime \prime}$. For any point $w \in \partial G^{\prime} \cap \partial G^{\prime \prime}$ there exists a function $u_{w} \in K_{q}$ such that $u_{w}(w)>M$ and, by the continuity of $u_{w}$, this inequality extends to some neighborhood $V_{w}$ of $w$. The complementary part of the boundary $G^{\prime} \backslash G^{\prime \prime}$ lies on the boundary of $G_{0}$ in the disk $B\left(z^{\prime}, r_{0}\right)$. Hence, for a point $\zeta$ in this part of the boundary, we have

$$
U_{q}(\zeta)=\ln M(q d(\zeta)) \geq v(\zeta)+2 .
$$

Hence, for each point $w$ in this part of the boundary there exists a function $u_{w} \in K_{q}$ satisfying $u_{w}(w)>v(w)+1$. Again, by the continuity of $u_{w}$ and $v$, this inequality extends to some neighborhood $V_{w}$ of $w$. Since $\partial\left(G^{\prime} \backslash G^{\prime \prime}\right)$ is a compact set, we can choose a finite subcovering $V_{w_{1}}, \ldots, V_{w_{m}}$ of the covering $\left\{V_{w}, w \in \partial\left(G^{\prime} \backslash G^{\prime \prime}\right)\right\}$, where $w_{1}, \ldots, w_{m} \in \partial\left(G^{\prime} \backslash G^{\prime \prime}\right)$. Put $u(z)=\max _{k=1, \ldots, m} u_{w_{k}}(z)$. Obviously, $u(z) \in K_{q}$ and, by construction, we have $u(z)>v(z)$ on the set $V=\bigcup V_{w_{k}}$. We introduce the function

$$
u_{0}(\zeta)= \begin{cases}\max (u(\zeta), v(\zeta)) & \text { if } z \notin G^{\prime} \backslash G^{\prime \prime}, \\ u(\zeta) & \text { if } \zeta \in G^{\prime} \backslash G^{\prime \prime} .\end{cases}
$$

Since in the neighborhood $V$ of the boundary $\partial\left(G^{\prime} \backslash G^{\prime \prime}\right)$ we have $u_{0}(\zeta)=u(\zeta)$, the function $u_{0}$ is subharmonic and continuous. Moreover, $u_{0} \in K_{q}$. The necessary inequalities on the set $G_{0}$ follow from the fact that both functions $u$ and $v$ satisfy these inequalities there. On the set $G^{\prime} \backslash G^{\prime \prime}$, the necessary inequalities follow because $u \in K_{q}$. Finally, in $G^{\prime \prime}$ we have

$$
v(\zeta) \leq M<M+1 \leq \ln M(q d(\zeta)) .
$$

Thus, $u_{0} \in K_{q}$, and so $u_{0}\left(z_{1}\right) \leq U_{q}\left(z_{1}\right)$.

On the other hand, $u_{0}\left(z_{1}\right) \geq v\left(z_{1}\right) \geq U_{q}\left(z_{1}\right)+2$, a contradiction.

Lemma 9 is proved.

Lemmas 7, 8, and 9 make it possible to state new quasianalyticity criteria.

Theorem 4. Let the sequence $\left(m_{n}\right)$ be regular, and let $z=0$ be on the boundary of a bounded Jordan domain $D$. Then the class $A(\bar{D}, \mathcal{M})$ is quasianalytic at the point $z=0$ if and only if

$$
\sup \left\{v(z), v \in K_{q}\right\}=\infty, \quad \zeta \in D,
$$

for any $q \in \mathbb{N}$.

Proof. If the assumptions of the theorem are fulfilled, then, by Lemma 8 , for any $q \in \mathbb{N}$ we have

$$
U_{q}(\zeta) \equiv \ln M(q d(\zeta)), \quad \zeta \in G
$$


By Theorem 3, in this case the class $A(\bar{D}, \mathcal{M})$ is quasianalytic. Conversely, if the class $A(\bar{D}, \mathcal{M})$ is quasianalytic, then we have $(3.2)$ by Theorem 3. By Lemma 9, the function $U_{q}$ cannot be finite in $D$, and therefore, the conclusion of Theorem 4 follows from Lemma 7.

Theorem 4 is proved.

Theorem 5. Let the sequence $\left(m_{n}\right)$ be regular, and let $z=0$ be on the boundary of a bounded Jordan domain $D$. Then the class $A(\bar{D}, \mathcal{M})$ is nonquasianalytic at the point $z=0$ if and only if, for any $q \in \mathbb{N}$ greater than some $q_{0}$, there is a domain $D_{q}$ containing $\bar{D} \backslash\{0\}$ and a function $h(\zeta)$ that is harmonic in $D_{q}$, equals $\ln M(q d(\zeta))$ on the boundary of $D_{q}$, and satisfies

$$
\lim _{|z| \longrightarrow 0} \frac{h(z)}{-\ln |z|}=+\infty
$$

Proof. Obviously, under the assumptions of the theorem, the class $A(\bar{D}, \mathcal{M})$ cannot be quasianalytic.

Assume that the class $A(\bar{D}, \mathcal{M})$ is nonquasianalytic. Then, for a fixed $q \in \mathbb{N}$, we extend the function $\ln M(q d(\zeta))$ to the entire plane assuming that it is equal $+\infty$ on $\bar{D}$. Let

$$
D^{\prime}=\left\{\zeta \in \bar{C}: U_{q}(\zeta)<\ln M(q d(\zeta))\right\} .
$$

Under our assumptions, by Theorem 4 , there exists $q_{0} \in \mathbb{N}$ such that $D^{\prime} \supset D$, and the intersection $D^{\prime} \cap G$ is nonempty by Lemma 9. It is clear that the same is true for $q>q_{0}$.

Lemma 10. The sets

$$
\begin{aligned}
G^{\prime} & =D^{\prime} \cap G=\left\{\zeta \in G: U_{q}(\zeta)<\ln M(q d(\zeta))\right\} \\
G^{\prime \prime} & =\left\{\zeta \in G: U_{q}^{*}(\zeta)<\ln M(q d(\zeta))\right\}
\end{aligned}
$$

coincide, are open in $G$, and the function $U_{q}(\zeta)$ is harmonic in $G^{\prime}$.

Proof. Clearly, $G^{\prime \prime} \subset G^{\prime}$. Assume that there is a point $\zeta_{0} \in G$ such that $\zeta_{0} \in G^{\prime} \backslash G^{\prime \prime}$. To simplify the notation, we put $T=\ln M\left(q d\left(\zeta_{0}\right)\right)$ and assume that

$$
U_{q}\left(\zeta_{0}\right)=(1-2 a) T
$$

for some $a \in(0,1 / 2)$. Take the largest $r>0$ such that the harmonic majorant $V(\zeta)$ of the function $\ln M(q d(\zeta))$ in the disk $B\left(\zeta_{0}, r\right)$ satisfies the condition

$$
V(\zeta) \leq \ln M(q d(\zeta))+a T
$$

Now we fix an arbitrary $\epsilon>0$ and choose a point $w$ in $B\left(\zeta_{0}, r\right)$ with the property

$$
U_{q}(w)>(1-\epsilon) T \text {. }
$$

This is possible because $\zeta_{0} \notin G^{\prime \prime}$, and so $U_{q}^{*}\left(\zeta_{0}\right)=\ln M\left(q d\left(\zeta_{0}\right)\right)$. Also, let $\left|w-\zeta_{0}\right|=\delta r$; we may assume that $\delta<\frac{1}{3}$. There exists $v \in K_{q}$ such that

$$
v(w)>U_{q}(w)-\epsilon T \text {. }
$$

Let $\widetilde{v}$ denote the harmonic extension of $v$ to the disk $B\left(\zeta_{0}, r\right)$. Since $\widetilde{v} \leq V$ in that disk, we have, by the choice of the number $r$,

$$
\widetilde{v}(\zeta)-a T \leq \ln M(q d(\zeta)), \quad \zeta \in G,
$$

that is, $\widetilde{v}(\zeta)-a T \in K_{q}$, and moreover,

$$
\widetilde{v}(w)-a T \geq v(w)-a T \geq U_{q}(w)-\epsilon T-a T \geq(1-a-2 \epsilon) T .
$$

Therefore, $\widetilde{v}(w)-a T \geq(1-a-2 \epsilon) T$. 
Applying the Harnack inequality to this function in the disk $B(w,(1-\delta) r)$, we obtain

$$
\widetilde{v}\left(\zeta_{0}\right)-a T \geq \frac{(1-\delta) r-\delta r}{(1-\delta) r+\delta r}(\widetilde{v}(w)-a T) .
$$

The last two inequalities imply that

$$
\widetilde{v}\left(\zeta_{0}\right)-a T \geq(1-2 \delta)(1-a-2 \epsilon) T .
$$

Consequently,

$$
U_{q}\left(\zeta_{0}\right) \geq(1-a) T-(2 \epsilon+2 \delta-2 \delta a-4 \delta \epsilon) T .
$$

Recalling (3.4), we see that $a \leq 2 \epsilon+2 \delta-2 \delta a-4 \delta \epsilon$.

Letting $\delta$ and $\epsilon$ tend to zero, we obtain $a=0$. This means that $\zeta_{0} \notin G^{\prime}$, a contradiction. Thus, $G^{\prime}=G^{\prime \prime}$. Since $U_{q}^{*}$ is upper semicontinuous and $\ln M(q d(\zeta))$ is continuous, the set $G^{\prime \prime}$ is open. Therefore, $G^{\prime}$ is also open.

We prove that the function $U_{q}$ is harmonic in $G^{\prime \prime}$. Let $\zeta_{0} \in G^{\prime \prime}$. Since

$$
\lim _{r \rightarrow 0} \frac{1}{2 \pi} \int_{0}^{2 \pi} U_{q}^{*}\left(\zeta_{0}+r e^{i \varphi}\right) d \varphi=U_{q}^{*}\left(\zeta_{0}\right),
$$

for any $\epsilon>0$ there exists $r^{\prime}>0$ such that for $r \leq r^{\prime}$ we have

$$
\frac{1}{2 \pi} \int_{0}^{2 \pi} U_{q}^{*}\left(\zeta_{0}+r e^{i \varphi}\right) d \varphi \leq U_{q}^{*}\left(\zeta_{0}\right)+\epsilon .
$$

We choose $\epsilon>0$ so small that $U_{q}^{*}\left(\zeta_{0}\right)+3 \epsilon<\ln M\left(q d\left(\zeta_{0}\right)\right)$, extend the function $U_{q}^{*}$ harmonically to the disk $B\left(\zeta_{0}, r^{\prime}\right)$, and denote the resulting function on $\mathbb{C} \backslash\{0\}$ by $\widetilde{U}_{q}$. Observe that

$$
\widetilde{U}_{q}\left(\zeta_{0}\right)=\frac{1}{2 \pi} \int_{0}^{2 \pi} U_{q}^{*}\left(\zeta_{0}+r^{\prime} e^{i \varphi}\right) d \varphi \leq U_{q}^{*}\left(\zeta_{0}\right)+\epsilon .
$$

For $\delta \in(0 ; 1)$, by the Harnack inequality in the disk $B\left(\zeta_{0}, \delta r^{\prime}\right)$, we have

$$
\widetilde{U}_{q}(\zeta) \leq \frac{1+\delta}{1-\delta} \widetilde{U}_{q}\left(\zeta_{0}\right) \leq \frac{1+\delta}{1-\delta}\left(U_{q}^{*}\left(\zeta_{0}\right)+\epsilon\right)
$$

Thus, for sufficiently small $\delta>0$ in the disk $B\left(\zeta_{0}, \delta r^{\prime}\right)$ we have

$$
\widetilde{U}_{q}(\zeta) \leq U_{q}^{*}\left(\zeta_{0}\right)+2 \epsilon
$$

Let $r>0$ be so small that in the disk $B\left(\zeta_{0}, r\right)$ we have the inequality

$$
\ln M(q d(\zeta)) \geq \ln M\left(q d\left(\zeta_{0}\right)\right)-\epsilon .
$$

(Such a choice is possible by the continuity of $M(x)$.) Put $r_{0}=\min \left(\delta r^{\prime}, r\right)$. Then in the disk $B\left(\zeta_{0}, r_{0}\right)$ we have

$$
\widetilde{U}_{q}(\zeta) \leq\left(U_{q}^{*}\left(\zeta_{0}\right)+2 \epsilon\right) \leq \ln M\left(q d\left(\zeta_{0}\right)\right)-\epsilon \leq \ln M(q d(\zeta)) .
$$

Each $v$ in $K_{q}$ is less than or equal to $U_{q}^{*}$, so $v$ does not exceed $\widetilde{U}_{q}(\zeta)$ on the boundary of the disk $B\left(\zeta_{0}, r_{0}\right)$. If we extend $v$ harmonically to $B\left(\zeta_{0}, r_{0}\right)$, then the resulting function $\widetilde{v}$ will not exceed $\widetilde{U}_{q}(\zeta)$ in this disk by the maximum principle. By (3.5), all functions $\widetilde{v}$ obtained in this way are in the class $K_{q}$ and are harmonic in the disk under consideration. Then the function $U_{q}$ will also be harmonic in $B\left(\zeta_{0}, r_{0}\right)$ as an upper envelope of a bounded family of harmonic functions; see 12 .

It is obvious that relation (3.3) holds true for the function $U_{q}(z)$.

Lemma 10 is proved.

To clarify what happens to the points of the boundary of $D$, we need the following lemma concerning the properties of the function $M(x)$. 
Lemma 11. Let $p(x)$ denote the smallest natural number $p$ such that

$$
M(x)=\frac{1}{m_{p} x^{p}}
$$

such numbers exist by the definition of the function $M(x)$. Then

$$
\lim _{x \rightarrow 0} p(x)=\infty .
$$

Also,

$$
\lim _{x \rightarrow 0} \frac{\ln M(x)}{-\ln x}=\infty
$$

Proof. To prove the first statement, assume the contrary. Suppose that for a sequence $x_{n} \rightarrow 0$ we have $p\left(x_{n}\right)<p$. Then

$$
\frac{1}{m_{p\left(x_{n}\right)} x_{n}^{p\left(x_{n}\right)}} \geq \frac{1}{m_{p} x_{n}^{p}}
$$

and, for $x_{n}<1$,

$$
x_{n}>x_{n}^{p-p\left(x_{n}\right)} \geq \frac{\min \left\{m_{k}, k=0,1, \ldots, p-1\right\}}{m_{p}} .
$$

Let $n$ tend to infinity. Then either $\min \left\{m_{k}, k=0,1, \ldots, p-1\right\}=0$ or $m_{p}=\infty$, which is impossible.

Now we take $j \in \mathbb{N}$ and let $1>\delta>0$ be so small that $p(x)>j$ for all $x<\delta$. Then

$$
\ln M(x)=\ln \frac{1}{m_{p(x)} x^{p(x)}} \geq-\ln m_{j}-j \ln x, \quad x<\delta .
$$

Hence,

$$
\lim _{x \rightarrow 0} \frac{\ln M(x)}{-\ln x} \geq j .
$$

Lemma 11 is proved.

Now we study the points on the boundary of $D$.

Lemma 12. The set $\partial D \backslash\{0\}$ is contained in $D^{\prime}$; the function $U_{q}$ is harmonic at the points of this set and satisfies (3.3).

Proof. First, we prove that on $\partial D$ there are points where $U_{q}$ is locally bounded. It is clear that such points belong to $D^{\prime}$. We fix an arbitrary point $z \in \partial D$ and some number $\rho>0$. By property (1.3) of regular sequences, there exists a number $p_{0}=p(\rho)$ such that for all $p \geq p_{0}$ we have

$$
m_{p} \rho^{p}>1,
$$

whereas, by Lemma 11, there exists $\delta>0$ such that $p(x)>p_{0}$ for all $x \in(0 ; \delta)$. By Lemma 9, all boundary points are limit points for $G^{\prime}$. Take a point $w \in G^{\prime}$ in the disk $B(z, \min (\rho, \delta))$. Let $\epsilon>0$ be such that

$$
U_{q}(w) \leq \ln M(q d(w))-2 \epsilon,
$$

and let $f_{w}(\zeta)$ be a function of the form $1 / m_{p}(\zeta-t)^{p}$, where $t \in D, p \in \mathbb{N}$; this function satisfies the estimates

$$
\ln \left|f_{w}(\zeta)\right| \leq \ln M(q d(\zeta)), \quad \zeta \in G ; \quad \ln \left|f_{w}(w)\right| \geq \ln M(q d(w))-\epsilon .
$$

Since $d(w)<\delta$, the number $p$ in the formula for $f_{w}$ is at least $p_{0}$, and, by the choice of $p_{0}$,

$$
\ln \left|f_{w}(\zeta)\right|<1, \quad \zeta \notin B(t, \rho) .
$$


Let $v \in K_{q}$ be an arbitrary function, and denote by $V$ the connected component of the set

$$
\left\{\zeta: v(\zeta)<\ln \left|f_{w}(\zeta)\right|\right\}
$$

that contains the point $w$. Should the singularity point $t$ be situated outside $V$, the function

$$
\widetilde{v}(\zeta)= \begin{cases}\max \left(v(\zeta), \ln \left|f_{w}(\zeta)\right|\right) & \text { if } \zeta \in V, \\ v(\zeta) & \text { if } \zeta \notin V,\end{cases}
$$

would belong to the class $K_{q}$, which leads to the estimate

$$
U_{q}(w) \geq \widetilde{v}(w)=\ln \left|f_{w}(w)\right| \geq \ln M(q d(w))-\epsilon,
$$

and this contradicts (3.6). Thus, the component $V$ contains the singularity point $t$ of $f_{w}$. Note that, since $v \geq 0$, the component $V$ is in $B(t, \rho)$ by (3.7). Thus, the point $w$ can be connected with the point $t$ by a path in $B(t, \rho)$ on which we have

$$
v(\zeta)<\ln \left|f_{w}(\zeta)\right| \text {. }
$$

Let $2 \sigma=\inf \{|t-\zeta|, \zeta \in \partial D\}$, and let $t_{0}$ be the first point of the circle $|t-\zeta|=\sigma$ lying on this path (if we start from $w$ ). On the part of the path from $w$ to $t_{0}$ we have

$$
v(\zeta)<\ln \left|f_{w}(\zeta)\right| \leq \frac{1}{m_{p} \sigma^{p}}
$$

Note that the number $1 / m_{p} \sigma^{p}$ does not depend on the function $v$.

Now, we take two points $z_{1}, z_{2} \in \partial D$ and put $\rho=\left|z_{1}-z_{2}\right| / 3$. Repeating the above constructions for each of these points, we find points $w_{i} \in G^{\prime}, t_{i} \in D$ for the points $z_{i}$, $i=1,2$, such that for any function $v \in K_{q}$ there is a path $\gamma_{i} \subset B\left(t_{i}, \rho\right)$ connecting $w_{i}$ and $t_{i}$, and on each of these two paths we have

$$
v(\zeta) \leq M_{i},
$$

where $M_{i}$ does not depend on $v$. Put

$$
s=\min \left(d\left(w_{1}\right), d\left(w_{2}\right), \inf _{\zeta \in \partial D}\left|t_{1}-\zeta\right|, \inf _{\zeta \in \partial D}\left|t_{2}-\zeta\right|\right) .
$$

We connect the points $w_{1}, w_{2}$ by a path $l_{1}$ in the domain $\{\zeta: d(\zeta)>s / 2\}$, and the points $t_{1}, t_{2}$, by a path $l_{2}$ in $D$ at the distance of at least $s / 2$ from $\partial D$. Let $M^{\prime}=\max \left\{U_{q}(\zeta), \zeta \in l_{2}\right\}$. Then on the contour composed of $\gamma_{i}, l_{i}, i=1,2$, we have the estimate

$$
v(\zeta) \leq \max \left(M_{1}, M_{2}, M^{\prime}, \ln M\left(\frac{q s}{2}\right)\right)=M .
$$

By the maximum principle, this estimate extends to the interior $V_{0}$ of the contour. In particular, this estimate holds true on the set $V_{0} \backslash\left(B\left(t_{1}, \rho\right) \cup B\left(t_{2}, \rho\right)\right)$, which does not depend on the function $v$. Since $\left|t_{1}-t_{2}\right| \geq\left|z_{1}-z_{2}\right|-\left|z_{1}-t_{1}\right|-\left|z_{2}-t_{2}\right|>3 \rho$, this set is nonempty. Hence, $U_{q}(\zeta) \leq M$ on this set.

Thus, we have found points on $\partial D$ where $U_{q}$ is locally bounded.

We prove that the set of points on $\partial D$ where $U_{q}$ is locally bounded is connected. Let $z, w \in \partial D$, and let the function $U_{q}$ be bounded in $B\left(z, r_{1}\right), B\left(w, r_{2}\right)$. Consider the diameter $d_{1}$ of the disk $B\left(z, r_{1}\right)$ with endpoints $z_{1} \in D, z_{2} \in G$ and the diameter $d_{2}$ of the disk $B\left(w, r_{2}\right)$ with endpoints $w_{1} \in D, w_{2} \in G$. We connect the points $z_{1}, w_{1}$ by a continuous path $l_{1}$ in $D$ and the points $z_{2}, w_{2}$, by a continuous path $l_{2}$ in $G$. Put

$$
\begin{aligned}
C_{1} & =\max \left\{U_{q}(\zeta), \quad \zeta \in d_{1} \cup l_{1} \cup d_{2}\right\}, \\
\epsilon & =\min \left\{d(\zeta), \zeta \in l_{2}\right\}, \\
C & =\max \left(C_{1}, \ln M(q \epsilon)\right) .
\end{aligned}
$$


Obviously, on the contour composed of the diameters $d_{1}, d_{2}$ and the paths $l_{1}, l_{2}$, we have $U_{q}(\zeta) \leq C$. By the maximum principle, this estimate extends to the domain bounded by the contour. Hence, the function $U_{q}$ is locally bounded at the points of the arc of the boundary of $D$ between $z$ and $w$.

Now we show that $U_{q}$ is locally bounded at all points of the set $\partial D \backslash\{0\}$. Let $\Gamma_{0}$ be the maximal connected arc of the boundary such that $U_{q}$ is locally bounded on that arc and $U_{q}$ is not locally bounded at some boundary point $z \neq 0$. Assume that

$$
2 \rho=\inf _{\zeta \in \Gamma_{0}}|z-\zeta|>0
$$

and perform the above construction for the point $z$ with a given $\rho$; as a result, we find points $w \in G^{\prime}, t \in D$ such that for any function $v \in K_{q}$ there is a path $\gamma$ in $B(t, \rho)$ that connects the points and the estimate

$$
v(\zeta) \leq M
$$

holds true for all points of the path, where $M$ does not depend on $v$. Connect the points $w$ and $t$ by a continuous curve $\gamma_{0}$ such that it intersects the boundary of $D$ at a point of the $\operatorname{arc} \Gamma_{0}$. Obviously, this curve can be chosen so that the function $U_{q}$ is locally bounded on it. On the contour composed of the curves $\gamma$ and $\gamma_{0}$ the function $v$ will be bounded by a constant independent of $v$. Extending this estimate inside by the maximum principle, we conclude that $v$ is bounded on the interior of the contour. This interior $V_{0}$ depends on the function $v$, but its nonempty subset $V_{0} \backslash B(t, \rho)$ does not depend on $v$. Hence, we see that $U_{q}$ is bounded on $V_{0} \backslash B(t, \rho)$. By the choice of $\rho$, the latter set contains a boundary arc that is not contained in $\Gamma_{0}$, which contradicts the maximality of $\Gamma_{0}$.

Thus, the function $U_{q}$ is locally bounded on the set $\partial D \backslash\{0\}$. Let $z$ be an arbitrary point in this set. There exist constants $M, r>0$ such that

$$
U_{q}(\zeta) \leq M \leq \ln M(q d(\zeta)), \quad \zeta \in B(z, r) .
$$

Replacing each function $v \in K_{q}$ by its harmonic extension to the disk $B(z, r)$, we obtain a right-directed family of functions harmonic in $B(z, r)$, and $U_{q}$ will be harmonic in $B(z, r)$, as the upper envelope of this family.

Relation (3.3) holds true for the function $U_{q}$ by its definition.

Lemma 12 is proved.

To complete the proof of Theorem 5 , it remains to show that the set $D^{\prime}$ is connected. Let $w \in G^{\prime}$, and let $\epsilon>0$ be such that

$$
U_{q}(w) \leq \ln M(q d(w))-2 \epsilon .
$$

Again, we use a function $f_{w}(\zeta)$ of the form $1 / m_{p}\left(\zeta-z_{0}\right)^{p}$, where $p \in \mathbb{N}, z_{0} \in D$, that satisfies

$$
\ln \left|f_{w}(\zeta)\right| \leq \ln M(q d(\zeta)), \quad \zeta \in G, \quad \ln \left|f_{w}(w)\right| \geq \ln M(q d(w))-\epsilon .
$$

Let $V$ be the connected component of the set

$$
\left\{\zeta: U_{q}(\zeta)<\ln \left|f_{w}(\zeta)\right|\right\}
$$

that contains the point $w$. Should the singularity point $z_{0}$ not belong to $V$, the harmonic functions $U_{q}$ and $\ln \left|f_{w}\right|$ would coincide on the boundary $V$ and therefore in $V$, in particular, at the point $w$. But this cannot be true because

$$
\ln \left|f_{w}(w)\right| \geq \ln M(q d(w))-\epsilon \geq U_{q}(w)+\epsilon .
$$

Hence, $z_{0} \in V$, and this point can be connected with $w$ by a path in $V \subset D^{\prime}$. This implies that the set $D^{\prime}$ is connected.

Theorem 5 is proved. 


\section{§4. LOCALIZATION OF THE QUASIANALYTICITY PROBLEM}

In this section we prove that the quasianalyticity problem is local: if two domains $D_{1}$ and $D_{2}$ coincide in a neighborhood of a common boundary point $z_{0}$, then the classes $A\left(\bar{D}_{1}, \mathcal{M}\right)$ and $A\left(\bar{D}_{2}, \mathcal{M}\right)$ are simultaneously quasianalytic or nonquasianalytic at $z_{0}$.

By using criteria already known, we can deduce new quasianalyticity criteria from this property under certain restrictions on the domain.

Theorem 6. Let the sequence $\left(m_{n}\right)$ be regular, and let $z=0$ be a common boundary point of two bounded Jordan domains $D^{\prime}$ and $D^{\prime \prime}$. If for some $r>0$ these two domains coincide in the disk $B(0,2 r)$, i.e.,

$$
D^{\prime} \cap B(0,2 r)=D^{\prime \prime} \cap B(0,2 r),
$$

then the classes $A\left(\bar{D}^{\prime}, \mathcal{M}\right)$ and $A\left(\bar{D}^{\prime \prime}, \mathcal{M}\right)$ are simultaneously quasianalytic or nonquasianalytic at the point $z=0$.

Proof. We need an auxiliary lemma.

Lemma 13. Let $z=0$ be a common boundary point of two bounded Jordan domains $D^{\prime}$ and $D^{\prime \prime}$. If for some $r>0$ these two domains coincide in the disk $B(0,2 r)$, i.e.,

$$
D^{\prime} \cap B(0,2 r)=D^{\prime \prime} \cap B(0,2 r),
$$

then there exist a positive number $p$ and a Jordan domain $D$ such that

a) $D \subset D^{\prime} \cap B(0,2 r)=D^{\prime \prime} \cap B(0,2 r)$ and the boundary of $D$ lies on the circle $|z|=2 r$ and on the common part of the boundaries of $D^{\prime}$ and $D^{\prime \prime}$;

b) for $\zeta \notin D$ and $|\zeta| \leq p$, the distance from $\zeta$ to the boundary of $D$ coincides with the distance from $\zeta$ to the boundaries of $D^{\prime}$ and $D^{\prime \prime}$ :

$$
d(\zeta):=\inf _{z \in D}|z-\zeta|=\inf _{z \in D^{\prime}}|z-\zeta|=\inf _{z \in D^{\prime \prime}}|z-\zeta|
$$

Proof. Let $D$ be the connected component of the intersection $D^{\prime} \cap B(0,2 r)$ that has the point $z=0$ on its boundary. By assumptions, $D$ coincides with the corresponding part of the intersection $D^{\prime \prime} \cap B(0,2 r)$. Obviously, the domain $D$ is simply connected. Let $z=z(t),|t| \leq 1$, be a continuous parametrization of the boundary of $D^{\prime}$ satisfying $z(0)=0$. We denote by $I$ the set of points $t \in[-1 ; 1]$ for which $|z(t)|<2 r$. Let $(\alpha ; \beta)$ be the largest interval in $I$ containing the point $t=0$. Put

$$
p=\frac{1}{2} \inf \{|z(t)|, t \notin(\alpha ; \beta)\} .
$$

Since the boundary of $D$ is a Jordan curve without self-intersections, it follows that $p>0$. For any point $\zeta \in B(0, p) \backslash D$, we have

$$
d(\zeta)=\inf _{z \in D}|z-\zeta| \leq|\zeta|<p<\inf \{|z(t)|, t \notin(\alpha ; \beta)\}
$$

i.e.,

$$
d(\zeta)=\inf \{|z(t)|, t \in(\alpha ; \beta)\} \geq \inf \{|z(t)|, t \in[-1 ; 1]\}=d^{\prime}(\zeta) .
$$

Consequently, for $\zeta \in B(0, p) \backslash D$ we have

$$
d(\zeta)=d^{\prime}(\zeta):=\inf _{z \in D^{\prime}}|z-\zeta|
$$

Since the domains $D^{\prime}$ and $D^{\prime \prime}$ coincide in the disk $B(0, p)$, for $\zeta$ as above we have

$$
d(\zeta)=d^{\prime \prime}(\zeta):=\inf _{z \in D^{\prime \prime}}|z-\zeta| .
$$

Lemma 13 is proved. 
Obviously, it suffices to prove simultaneous quasianalyticity for the classes $A\left(\bar{D}^{\prime}, \mathcal{M}\right)$ and $A(\bar{D}, \mathcal{M})$ at the point $z=0$. Denote by $G, G^{\prime}$ the complements of $\bar{D}, \bar{D}^{\prime}$, respectively, to the extended complex plane, i.e., $G=\overline{\mathbb{C}} \backslash \bar{D}, G^{\prime}=\overline{\mathbb{C}} \backslash \bar{D}^{\prime}$. Let $d(\zeta), d^{\prime}(\zeta)$ be the distances from the point $\zeta$ to the domains $D, D^{\prime}$. These function are defined on the domains $G$ and $G^{\prime}$, respectively. By Lemma 13 ,

$$
G \cap B(0, p)=G^{\prime} \cap B(0, p),
$$

and also $d(\zeta)=d^{\prime}(\zeta)$ for $\zeta \in G \cap B(0, p)$.

Since $D \subset D^{\prime}$, it follows that $d^{\prime}(\zeta) \geq d(\zeta)$ for $\zeta \in G^{\prime}$, whence

$$
M(q d(\zeta)) \leq M\left(q d^{\prime}(\zeta)\right)
$$

for any $q \in \mathbb{N}$.

Assume that the class $A(\bar{D}, \mathcal{M})$ is quasianalytic. By Theorem 4 , for any $q \in \mathbb{N}$ the relation

$$
\sup \left\{v(z), v \in K_{q}(D)\right\}=\infty, \quad \zeta \in D,
$$

is fulfilled. By property (4.1), we have the inclusion $K_{q}(D) \subset K_{q}\left(D^{\prime}\right)$, and so

$$
\sup \left\{v(z), v \in K_{q}\left(D^{\prime}\right)\right\}=\infty, \quad \zeta \in D .
$$

By Theorem 4 , the class $A\left(\bar{D}^{\prime}, \mathcal{M}\right)$ is also quasianalytic.

Now, assume that the class $A(\bar{D}, \mathcal{M})$ is nonquasianalytic. By Theorem 5 , for any $q \in \mathbb{N}$ starting with some $q_{0}$, there is a domain $D_{q}$ containing $\bar{D} \backslash\{0\}$, and a function $h(\zeta)$ harmonic in $D_{q}$ that is equal to $\ln M(q d(\zeta))$ on the boundary and satisfies

$$
\lim _{|\zeta| \longrightarrow 0} \frac{h(\zeta)}{-\ln |\zeta|}=+\infty
$$

Theorem 5 means that, for any fixed $q \in \mathbb{N}, q \geq q_{0}$, the function

$$
u(z)=\sup \left\{v(z), v \in K_{q}(D)\right\}, \quad z \in D,
$$

is well defined and subharmonic on the extended plane except for the point zero, i.e., on $\overline{\mathbb{C}} \backslash\{0\}$, and is harmonic in the domain $D_{q}$, containing $\bar{D} \backslash\{0\}$. The domain $D^{\prime}$ is bounded and the ratio $|\zeta| / d^{\prime}(\zeta)$ tends to 1 as $|\zeta| \longrightarrow+\infty$. Therefore, there exists a sufficiently large number $R$ such that

$$
D^{\prime} \subset B(0, R), \quad \frac{|\zeta|}{d^{\prime}(\zeta)} \leq 2 \quad \text { for } \quad|\zeta| \geq R
$$

Let $C$ denote the open annulus bounded by the circles $|z|=p$ and $|z|=R$. Let the Borel measure $\mu$ in the domain $\overline{\mathbb{C}} \backslash\{0\}$ be the Riesz measure associated with the subharmonic function $u(\zeta)$, and let $\mu_{0}$ be the restriction of the measure $\mu$ to the ring $\bar{C}$. Since $\bar{C}$ is a compact set in $\overline{\mathbb{C}} \backslash\{0\}$, it follows that $\mu_{0}(\bar{C})<\infty$ and the logarithmic potential of the measure $\mu_{0}$,

$$
u_{0}(\zeta)=\int \ln |\zeta-z| d \mu_{0}(z)
$$

is well defined and subharmonic on the entire plane $\mathbb{C}$ and harmonic on $\mathbb{C} \backslash C$. The difference $u(\zeta)-u_{0}(\zeta)$ is harmonic on the union $\left(C \cup D_{q}\right)$, and, in particular, in the domain

$$
\Omega=\left(C \cup D_{q}\right) \cap B(0, R) .
$$

The boundary of $\Omega$ consists of the circle $|\zeta|=R$ and of a part $\gamma$ lying inside the circle $\bar{B}(0, p)$. By the choice of the numbers $p$ and $R$, we have

$$
\begin{aligned}
\ln M\left(2 q d^{\prime}(\zeta)\right) & \leq \ln M(q|\zeta|), \quad|\zeta| \geq R, \\
\ln M\left(2 q d^{\prime}(\zeta)\right)= & \ln M(2 q d(\zeta)) \leq \ln M(q d(\zeta)), \quad|\zeta| \leq p, \quad \zeta \in G^{\prime} .
\end{aligned}
$$


Take an arbitrary function $v(\zeta)$ in the class $K_{2 q}\left(D^{\prime}\right)$. Then $v$ is nonnegative, continuous, and subharmonic on $\overline{\mathbb{C}} \backslash\{0\}$ and satisfies the estimates

$$
v(\zeta)=O\left(\ln \frac{1}{|\zeta|}\right) \quad \text { as } \zeta \rightarrow 0 ; \quad v(\zeta) \leq \ln M\left(2 q d^{\prime}(\zeta)\right), \quad \zeta \in G^{\prime}
$$

Using (4.2), from the second inequality we deduce that

$$
\begin{aligned}
& v(\zeta) \leq \ln M(q|\zeta|), \quad|\zeta| \geq R, \\
& v(\zeta) \leq \ln M(q d(\zeta)), \quad|\zeta| \leq p, \quad \zeta \in G^{\prime} .
\end{aligned}
$$

Note that the functions $\max \left(-\ln m_{k}-k \ln q|\zeta|, 0\right)$ are subharmonic, nonnegative, and continuous on the extended plane except for zero, and also

$$
\max \left(-\ln m_{k}-k \ln q|\zeta|, 0\right) \leq \ln M(q d(\zeta)), \quad \zeta \notin D,
$$

that is, these functions belong to $K_{q}(D)$. Hence, by the definition of the function $u(\zeta)$, we have $\ln M(q|\zeta|) \leq u(\zeta)$ for all $\zeta$, whence $v(\zeta) \leq u(\zeta),|\zeta| \geq R$.

Since

$$
B(0, p) \cap G^{\prime}=B(0, p) \cap G
$$

and the boundary of $\Omega$ coincides with the boundary of $D_{q}$ in $B(0, p)$, and on the boundary of $D_{q}$ the function $u(\zeta)$ equals $\ln M(q d(\zeta))$, we conclude that

$$
v(\zeta) \leq \ln M(q d(\zeta))=u(\zeta), \quad \zeta \in \partial \Omega \cap B(0, p) .
$$

Thus, we have $v(\zeta) \leq u(\zeta), \zeta \in \partial \Omega$.

The function $u_{0}(\bar{\zeta})$ is subharmonic in the entire plane, and therefore, is bounded in the disk $B(0, R)$ :

$$
u_{0}(\zeta) \leq T
$$

Hence, on the boundary of $\Omega$ we have $v(\zeta) \leq u(\zeta)-u_{0}(\zeta)+u_{0}(\zeta) \leq u(\zeta)-u_{0}(\zeta)+T$, $\zeta \in \partial \Omega$.

The function $u(\zeta)-u_{0}(\zeta)$ is harmonic in $\Omega$, and $v(\zeta)$ is subharmonic in this domain. By the maximum principle,

$$
v(\zeta) \leq u(\zeta)-u_{0}(\zeta)+T, \quad \zeta \in \Omega
$$

Being subharmonic on $\mathbb{C} \backslash\{0\}$, the function $u(\zeta)-u_{0}(\zeta)$ is bounded from above in the annulus $\left\{\frac{p}{2} \leq|\zeta| \leq R\right\}, u(\zeta)-u_{0}(\zeta) \leq T_{1}$.

By definition, the function $u(\zeta)$ is nonnegative; hence, the function $-u_{0}(\zeta)$ is also bounded from above in the same annulus,

$$
-u_{0}(\zeta) \leq T_{1}
$$

Since the function $u_{0}(\zeta)$ is harmonic in the disk $B(0, p)$, the function $-u_{0}(\zeta)$ is bounded from above in the disk $B\left(0, \frac{p}{2}\right)$ :

$$
-u_{0}(\zeta) \leq T_{2}
$$

Put $T^{\prime}=\max \left(T_{1}, T_{2}\right)$. Then the last two inequalities imply that the function $-u_{0}(\zeta)$ is bounded by $T^{\prime}$ in the disk $B(0, R)$. In particular, this estimate is valid for the domain $\Omega$ :

$$
-u_{0}(\zeta) \leq T^{\prime}, \quad \zeta \in \Omega
$$

Combined with (4.3), this inequality shows that $v(\zeta) \leq u(\zeta)+T^{\prime}+T, \zeta \in \Omega$.

Since $v$ is an arbitrary function in $K_{2 q}\left(D^{\prime}\right)$, the condition of Theorem 4 cannot be satisfied, and the class $A\left(\bar{D}^{\prime}, \mathcal{M}\right)$ cannot be quasianalytic.

Theorem 6 is proved. 
Theorem 7. Let $D, D_{1}$ be simply connected domains, let $\Omega$ be a domain containing the closure $\bar{D}$, and let $\varphi$ be an analytic function in $\Omega$ such that $\varphi(D) \subset D_{1}$. If the boundary point $w_{0} \in \partial D_{1}$ is the image of a boundary point $z_{0} \in \partial D$, i.e., $w_{0}=\varphi\left(z_{0}\right)$, then for any sequence $\mathcal{M}=\left(M_{n}\right)$ we have the inclusion

$$
\left\{f(\varphi(z)), f \in A\left(\bar{D}_{1}, \mathcal{M}\right)\right\} \subset A(\bar{D}, \mathcal{M}) .
$$

Proof. Let $f \in A\left(\bar{D}_{1}, \mathcal{M}\right)$. By Dyn'kin's theorem [1] (see Theorem B of the present paper), there exists a continuously differentiable function $F$ on $\mathbb{C}$ such that $F(w) \equiv f(w)$ in $D_{1}$ and

$$
\left|\frac{\partial F(w)}{\partial \bar{w}}\right| \leq \frac{C}{M\left(B d_{1}(w)\right)}, \quad w \in \mathbb{C},
$$

where $C, B$ are some positive constants, and $d_{1}(w)$ denotes the distance from the point $w \notin D_{1}$ to the boundary of $D_{1}$. Let $3 r=\inf \{|z-w|, z \in \bar{D}, w \notin \Omega\}$ be the distance from $\bar{D}$ to the boundary of $\Omega$. By the assumptions of the theorem, we have $r>0$. We denote by $\Omega^{\prime}$ and $\Omega^{\prime \prime}$ the $r$-envelope and the $2 r$-envelope of the set $\bar{D}$, respectively, i.e., $\Omega^{\prime}=\bigcup_{z \in D_{1}} B(z, r), \quad \Omega^{\prime \prime}=\bigcup_{z \in D_{1}} B(z, 2 r)$.

We take a smooth Jordan curve $\Gamma$ in $\Omega^{\prime \prime} \backslash \Omega^{\prime}$ that encloses the set $\bar{D}$ and denote by $\Omega_{1}$ the interior of the curve. Applying the Borel-Pompeiu formula (see [13]) to the function $g(z)=F(\varphi(z))$ in the domain $\Omega_{1}$, we obtain

$$
g(z)=\frac{1}{2 \pi i} \int_{\Gamma} \frac{g(t)}{t-z} d t-\frac{1}{\pi} \int_{\Omega_{1}} \frac{\partial g}{\partial \bar{t}} \frac{d v(t)}{t-z} .
$$

We prove that each term on the right-hand side of this identity belongs to the class $A(\bar{D}, \mathcal{M})$. Put

$$
u(z)=\frac{1}{2 \pi i} \int_{\Gamma} \frac{g(t)}{t-z} d t, \quad v(z)=\frac{1}{\pi} \int_{\Omega_{1}} \frac{\partial g}{\partial \bar{t}} \frac{d v(t)}{t-z} .
$$

For $z, \zeta \in D$ and $k, n \in \mathbb{N}, n \geq k$, we have

$$
\begin{aligned}
\mid u^{(k)}(\zeta) & -\sum_{p=0}^{n-k} u^{(k+p)}(z) \frac{(\zeta-z)^{p}}{p !} \mid \\
& =\frac{1}{2 \pi} \int_{\Gamma}|g(t)|\left|\frac{k !}{(t-z)^{k+1}}-\sum_{p=0}^{n-k} \frac{(p+k) !(\zeta-z)^{p}}{p !(t-z)^{p+k+1}}\right||d t| .
\end{aligned}
$$

We apply formula (1.7) and observe that if $z, \zeta \in D$ and $t \in \Gamma$, then $|\zeta-t|,|z-t| \geq r$. Therefore,

$$
\begin{aligned}
\mid u^{(k)}(\zeta) & -\sum_{p=0}^{n-k} u^{(k+p)}(z) \frac{(\zeta-z)^{p}}{p !} \mid \\
& \leq|\zeta-z|^{n-k+1} \frac{1}{2 \pi r^{n+2}} \max _{t \in \Gamma}|g(t)||\Gamma| \sum_{s=0}^{k} \frac{\left(\begin{array}{l}
k \\
s
\end{array}\right)(n-s) ! s !}{(n-k) !} \\
& =\max _{t \in \Gamma}|g(t)||\Gamma||\zeta-z|^{n-k+1} \frac{k !}{2 \pi r^{n+2}} \sum_{s=0}^{k}\left(\begin{array}{l}
n-s \\
k-s
\end{array}\right)
\end{aligned}
$$


where $|\Gamma|$ is the length of the curve $\Gamma$. The sum of binomial coefficients was evaluated in (1.10); this yields

$$
\begin{aligned}
\mid u^{(k)}(\zeta) & -\sum_{p=0}^{n-k} u^{(k+p)}(z) \frac{(\zeta-z)^{p}}{p !} \mid \\
& \leq \frac{1}{2 \pi} \max _{t \in \Gamma}|g(t)||\Gamma||\zeta-z|^{n-k+1} \frac{(n+1) !}{(n-k+1) ! r^{n+2}} .
\end{aligned}
$$

Property (1.3) of regular sequences shows that there exists a number $\delta>0$ such that $m_{n}^{\frac{1}{n}} \geq \delta, n=0,1, \ldots$, or $M_{n} \geq n ! \delta^{n}, n=0,1, \ldots$ Hence,

$$
\begin{aligned}
\mid u^{(k)}(\zeta) & -\sum_{p=0}^{n-k} u^{(k+p)}(z) \frac{(\zeta-z)^{p}}{p !} \mid \\
& \leq \frac{1}{2 \pi} \max _{t \in \Gamma}|g(t)||\Gamma||\zeta-z|^{n-k+1} \frac{M_{n+1}}{(n-k+1) ! \delta^{n+1} r^{n+2}} .
\end{aligned}
$$

Thus, we have obtained the estimate

$$
\begin{aligned}
& \sup _{n \geq 0, k \leq n} \sup _{z, \zeta \in D} \frac{(\delta r)^{n+1}(n-k+1) !}{M_{n+1}|z-\zeta|^{n-k+1}}\left|u^{(k)}(\zeta)-\sum_{p=0}^{n-k} u^{(k+p)}(z) \frac{(\zeta-z)^{p}}{p !}\right| \\
& \quad \leq \frac{\max _{t \in \Gamma}|g(t)||\Gamma|}{2 \pi r} .
\end{aligned}
$$

Next, we work with the function $v(z)$. Since, by the properties of the function $F(w)$, the integral in the definition of $v(z)$ is only taken over the domain $\Omega_{1} \backslash D$, for $z, \zeta \in D$ and $k, n \in \mathbb{N}, n \geq k$, we have

$$
\begin{aligned}
\mid v^{(k)}(\zeta) & -\sum_{p=0}^{n-k} v^{(k+p)}(z) \frac{(\zeta-z)^{p}}{p !} \mid \\
& \leq \frac{1}{\pi} \int_{\Omega_{1} \backslash D}\left|\frac{\partial g(t)}{\partial \bar{t}}\right|\left|\frac{k !}{(t-z)^{k+1}}-\sum_{p=0}^{n-k} \frac{(p+k) !(\zeta-z)^{p}}{p !(t-z)^{p+k+1}}\right| d v(t) .
\end{aligned}
$$

Again, we apply formula (1.7) and observe that $|\zeta-t|,|z-t| \geq d(t)$ for $t \in \Omega_{1} \backslash D$ and $\zeta, z \in D$; this yields

$$
\begin{aligned}
\mid v^{(k)}(\zeta) & -\sum_{p=0}^{n-k} v^{(k+p)}(z) \frac{(\zeta-z)^{p}}{p !} \mid \\
& \leq \frac{1}{\pi} \int_{\Omega_{1} \backslash D}\left|\frac{\partial g(t)}{\partial \bar{t}}\right| \frac{|\zeta-z|^{n-k+1} k !}{d(t)^{n+2}} \sum_{s=0}^{n-k}\left(\begin{array}{c}
n-s \\
k-s
\end{array}\right) d v(t) .
\end{aligned}
$$

Recalling (1.10), we obtain

$$
\left|v^{(k)}(\zeta)-\sum_{p=0}^{n-k} v^{(k+p)}(z) \frac{(\zeta-z)^{p}}{p !}\right| \leq \frac{\left|\Omega_{1}\right|}{\pi} \sup _{\Omega_{1} \backslash D}\left|\frac{\partial g(t)}{\partial \bar{t}}\right| \frac{|\zeta-z|^{n-k+1}(n+1) !}{d(t)^{n+2}(n-k+1) !},
$$

where $\left|\Omega_{1}\right|$ is the area of the domain $\Omega_{1}$. Using the definition of the function $g(t)$ and relation (4.4), we get

$$
\left|\frac{\partial g(t)}{\partial \bar{t}}\right|=\left|\frac{\partial F(w)}{\partial \bar{w}}(\varphi(t)) \overline{\varphi^{\prime}(t)}\right| \leq \frac{C}{M\left(B d_{1}(\varphi(t))\right)} \max _{t \in \Omega_{1}}\left|\varphi^{\prime}(t)\right| .
$$


Let $t \in \Omega_{1} \backslash D$ and $d(t)=\left|t-t_{0}\right|$, where $t_{0} \in \partial D$. Then $\varphi\left(t_{0}\right) \in \bar{D}_{1}$. Also $\left|t-t_{0}\right|<2 r$ and $B\left(t_{0}, 2 r\right) \subset \bar{\Omega}^{\prime \prime}$, and therefore,

$$
d_{1}(\varphi(t)) \leq\left|\varphi(t)-\varphi\left(t_{0}\right)\right| \leq \max _{\bar{\Omega}^{\prime \prime}}\left|\varphi^{\prime}(z)\right|\left|t-t_{0}\right|=\max _{\bar{\Omega}^{\prime \prime}}\left|\varphi^{\prime}(z)\right| d(t) .
$$

We denote the final quantity $\max _{\bar{\Omega}^{\prime \prime}}\left|\varphi^{\prime}(z)\right|$ by $T$. Thus, for $t \in \Omega_{1} \backslash D$, we get the estimate

$$
d_{1}(\varphi(t)) \leq T d(t) .
$$

Substituting this in (4.7), and using the monotonicity of the function $M(x)$, we obtain

$$
\left|\frac{\partial g(t)}{\partial \bar{t}}\right|=\left|\frac{\partial F(w)}{\partial \bar{w}}(\varphi(t)) \overline{\varphi^{\prime}(t)}\right| \leq \frac{T C}{M(B T d(t))} .
$$

We plug this in (4.6):

$$
\frac{(n-k+1) !}{|\zeta-z|^{n-k+1}}\left|v^{(k)}(\zeta)-\sum_{p=0}^{n-k} v^{(k+p)}(z) \frac{(\zeta-z)^{p}}{p !}\right| \leq \frac{T C\left|\Omega_{1}\right|}{\pi} \sup _{t \in \Omega_{1} \backslash D} \frac{1}{M(B T d(t)) d(t)^{n+2}} .
$$

By the properties (1.5) and (1.2) of regular sequences, we have

$$
\begin{aligned}
\sup _{t \in \Omega_{1} \backslash D} \frac{1}{M(B T d(t)) d(t)^{n+2}} & \leq \sup _{x>0} \frac{1}{M(B T x) x^{n+2}}=(B T)^{n+2} m_{n+2} \\
& \leq B T(B T Q)^{n+1} m_{n+1}=B T(B T Q)^{n+1} \frac{M_{n+1}}{(n+1) !} .
\end{aligned}
$$

Hence,

$$
\frac{(n-k+1) !}{|\zeta-z|^{n-k+1}}\left|v^{(k)}(\zeta)-\sum_{p=0}^{n-k} v^{(k+p)}(z) \frac{(\zeta-z)^{p}}{p !}\right| \leq \frac{B T^{2} C\left|\Omega_{1}\right|}{\pi}(B T Q)^{n+1} M_{n+1} .
$$

Thus, we obtain

$$
\begin{aligned}
& \sup _{n \geq 0, k \leq n} \sup _{\zeta, z \in D} \frac{(n-k+1) !}{(B T Q)^{n+1} M_{n+1}|\zeta-z|^{n-k+1}}\left|v^{(k)}(\zeta)-\sum_{p=0}^{n-k} v^{(k+p)}(z) \frac{(\zeta-z)^{p}}{p !}\right| \\
& \quad \leq \frac{B T^{2} C\left|\Omega_{1}\right|}{\pi} .
\end{aligned}
$$

Since $g(t) \equiv u(t)+v(t)$, combining this estimate with (4.5) yields

$$
\sup _{n \geq 0, k \leq n} \sup _{\zeta, z \in D} \frac{P^{n+1}(n-k+1) !}{M_{n+1}|\zeta-z|^{n-k+1}}\left|g^{(k)}(\zeta)-\sum_{p=0}^{n-k} g^{(k+p)}(z) \frac{(\zeta-z)^{p}}{p !}\right| \leq C^{\prime},
$$

where

$$
P=\min (\delta r, B T Q), \quad C^{\prime}=\frac{\max _{t \in \Gamma}|g(t)||\Gamma|}{2 \pi r}+\frac{B T^{2} C\left|\Omega_{1}\right|}{\pi} .
$$

Thus, $g(t) \in A(\bar{D}, \mathcal{M})$. Theorem 7 is proved.

Corollary 1. Suppose $D, D_{1}$ are simply connected domains, the domains $\Omega, \Omega_{1}$ contain the closures $\bar{D}, \bar{D}_{1}$, respectively, and $\varphi$ is a conformal mapping of $\Omega$ onto $\Omega_{1}$ such that $\varphi(D)=\varphi\left(D_{1}\right)$. If a boundary point $w_{0} \in \partial D_{1}$ is the image of a boundary point $z_{0} \in \partial D$, i.e., $w_{0}=\varphi\left(z_{0}\right)$, then for any sequence $\mathcal{M}=\left(M_{n}\right)$, the class $A\left(\bar{D}_{1}, \mathcal{M}\right)$ is quasianalytic at the point $w_{0}$ if and only if the class $A(\bar{D}, \mathcal{M})$ is quasianalytic at the point $z_{0}$. 
Proof. Let the class $A\left(\bar{D}_{1}, \mathcal{M}\right)$ be nonquasianalytic at $w_{0}$, i.e., there is a nonzero function $f \in A\left(\bar{D}_{1}, \mathcal{M}\right)$ that vanishes at $w_{0}$ with all its derivatives. By Theorem 7 , the function $g(w)=f(\varphi(w))$ belongs to $A(\bar{D}, \mathcal{M})$ and vanishes at $z_{0}$ together with all its derivatives. Thus, the class $A(\bar{D}, \mathcal{M})$ also cannot be quasianalytic. The proof of the converse statement is similar.

Corollary 2. Let $B^{\prime}=B^{\prime}(a, R)$ be the exterior of the disk $B(a, R)$ in the extended complex plane, i.e., $B^{\prime}(a, R)=\overline{\mathbb{C}} \backslash B(a, R)$. Then, for any point $z_{0} \in \partial B^{\prime}$, a criterion of quasianalyticity for the class $A\left(\bar{B}^{\prime}, \mathcal{M}\right)$ at the point $z_{0}$ is given by the condition

$$
\int_{1}^{\infty} \frac{\ln T(r)}{r^{\frac{3}{2}}} d r=\infty
$$

where

is the trace function of the sequence $\mathcal{M}$.

$$
T(r)=\sup _{n \geq 0} \frac{r^{n}}{M_{n}}
$$

Proof. This follows immediately from Corollary 1 , because $\varphi(w)=\frac{R}{w-a}$ is a conformal map of $\overline{\mathbb{C}}$ onto itself and $B^{\prime}$ is mapped onto the unit disk.

In what follows we consider domains whose boundary coincides locally with the graph of some function $y=u(x),|x|<\delta$. Denote by $\Omega(u, \delta)$ the supergraph of $u(x)$ on the interval $(-\delta ;+\delta)$, i.e.,

$$
\Omega(u)=\{z=x+i y: y>u(x),|x|<\delta\} .
$$

Theorem 8. Suppose that a Jordan domain D coincides locally with the supergraph of some function $y=u(x),|x|<\delta$, with $u(0)=0$. This means that for some $r>0$, the sets $D \cap B(0, r)$ and $\Omega(u, \delta) \cap B(0, r)$ coincide. Suppose that for some $a>0$ we have

$$
|u(x)| \leq a x^{2}
$$

then the class $A(\bar{D}, \mathcal{M})$ is quasianalytic at the point $z=0$ if and only if condition (4.8) is satisfied.

Proof. For $|x| \leq \frac{1}{2 a}$, we have the inequalities

$$
\begin{aligned}
& u_{+}(x):=\frac{1}{2 a}-\sqrt{\frac{1}{4 a^{2}}-x^{2}}=\frac{x^{2}}{\frac{1}{2 a}+\sqrt{\frac{1}{4 a^{2}}-x^{2}}} \geq a x^{2}, \\
& u_{-}(x):=-\frac{1}{2 a}+\sqrt{\frac{1}{4 a^{2}}-x^{2}}=\frac{-x^{2}}{\frac{1}{2 a}+\sqrt{\frac{1}{4 a^{2}}-x^{2}}} \leq-a x^{2} .
\end{aligned}
$$

Hence, if $|x|<\delta_{1}:=\min \left(\delta, \frac{1}{2 a}\right)$, then

$$
u_{+}(x) \geq u(x), \quad u_{-}(x) \leq u(x),
$$

and the supergraph $\Omega\left(u_{+}, \delta_{1}\right)$ is contained in the supergraph $\Omega\left(u, \delta_{1}\right)$, and $\Omega\left(u_{-}, \delta_{1}\right)$ contains $\Omega\left(u, \delta_{1}\right)$. The supergraph $\Omega\left(u_{+}, \frac{1}{2 a}\right)$ contains the open disk $B\left(\frac{i}{2 a}, \frac{1}{2 a}\right)$ and the supergraph $\Omega\left(u_{-}, \frac{1}{2 a}\right)$ is contained in the complement $B^{\prime}$ of the closed disk $B\left(\frac{-i}{2 a}, \frac{1}{2 a}\right)$. Put $\varepsilon=\min \left(\delta_{1}, r\right)$. Now, the claim follows from the inclusions

$$
\begin{aligned}
& B\left(\frac{i}{2 a}, \frac{1}{2 a}\right) \cap B(0, \varepsilon) \subset \Omega\left(u_{+}\right) \cap B(0, \varepsilon) \\
& \quad \subset \Omega(u) \cap B(0, \varepsilon) \subset \Omega\left(u_{-}\right) \cap B(0, \varepsilon) \subset B^{\prime} \cap B(0, \varepsilon)
\end{aligned}
$$

and from Corollary 2 of Theorem 7.

Theorem 8 is proved. 


\section{REFERENCES}

[1] E. M. Dyn'kin, Pseudoanalytic continuation of smooth functions. Uniform scale, Mathematical Programming and Related Questions (Proc. Seventh Winter School, Drogobych, 1974), Theory of Functions and Functional Analysis, Tsentral. Èkonom.-Mat. Inst. Akad. Nauk SSSR, Moscow, 1976, pp. 40-73. (Russian) MR0587795 (58:28536)

[2] J. Hadamard, Sur le module maximum d'une fonction et de ses dérivées, C. R. Séances Soc. Math. France 42 (1914).

[3] T. Carleman, Les fonctions quasi analytiques, Paris, 1926.

[4] A. Ostrowski, Über quasianalytische Funktionen und Bestimmtheit asymptotischer Entwickelungen, Acta Math. 53 (1930), 181-266. MR.1555294

[5] S. Mandelbrojt, Séries adhérentes, régularisation des suites, applications, Gauthier-Villars, Paris, 1952. MR0051893 (14:542f)

[6] Baltasar R.-Salinas, Functions with null moments, Rev. Acad. Ci. Madrid 49 (1955), 331-368. (Spanish) MR0080174 (18:204e)

[7] B. I. Korenblyum, Quasianalytic classes of functions in a circle, Dokl. Akad. Nauk SSSR 164 (1965), no. 1, 36-39; English transl., Soviet Math. Dokl. 6 (1965), 1155-1158. MR0212199|(35:3074)

[8] R. S. Yulmukhametov, Quasi-analytical classes of functions in convex domains, Mat. Sb. (N.S.) 130 (172) (1986), no. 4, 500-519; English transl., Math. USSR-Sb. 58 (1987), no. 2, 505-523. MR0867340 (88a:30076)

[9] , Approximation of subharmonic functions and applications, Thesis for a Doctor's Degree, Mat. Inst. Akad. Nauk SSSR, Moscow, 1987. (Russian)

[10] J. Sebastião e Silva, Su certe classi di spazi localmente convessi importanti per le applicazioni, Rend. Mat. e Appl. (5) 14 (1955), 388-410. MR0070046 (16:1122c)

[11] N. Sibony, Approximation polynomiale pondérée dans un domaine d'holomorphie de $\mathbb{C}^{n}$, Ann. Inst. Fourier (Grenoble) 26 (1976), no. 2, 71-99. MR0430312 (55:3317)

[12] M. Brelot, Éléments de la théorie classique du potentiel, Centre Doc. Univ., Paris, 1959. MR0106366 (21:5099)

[13] A. V. Bitsadze, Foundations of the theory of analytic functions of a complex variable, "Nauka", Moscow, 1972. (Russian) MR0390183 (52:11009)

Department of Mathematics, Bashirir State University, 450074 Ufa, Russia

E-mail address: trounovkv@mail.ru

Department of Mathematics, Bashkir State University, 450074 Ufa, Russia

Received 15/AUG/2006

Translated by A. BARANOV 